\title{
Numerical Renormalization Group for Quantum Impurities in a Bosonic Bath
}

\author{
Ralf Bulla*, Hyun-Jung Lee*, Ning-Hua Tong*, and Matthias Vojta ${ }^{\dagger}$ \\ * Theoretische Physik III, Elektronische Korrelationen und Magnetismus, Institut für Physik, Universität Augsburg, D-86135 \\ Augsburg, Germany \\ ${ }^{\dagger}$ Institut für Theorie der Kondensierten Materie, Universität Karlsruhe, D-76128 Karlsruhe, Germany
}

(October 30, 2018)

\begin{abstract}
We present a detailed description of the recently proposed numerical renormalization group method for models of quantum impurities coupled to a bosonic bath. Specifically, the method is applied to the spin-boson model, both in the Ohmic and sub-Ohmic cases. We present various results for static as well as dynamic quantities and discuss details of the numerical implementation, e.g., the discretization of a bosonic bath with arbitrary continuous spectral density, the suitable choice of a finite basis in the bosonic Hilbert space, and questions of convergence w.r.t. truncation parameters. The method is shown to provide high-accuracy data over the whole range of model parameters and temperatures, which are in agreement with exact results and other numerical data from the literature.
\end{abstract}

PACS: 05.10.Cc (Renormalization Group methods), 05.30.Jp (Boson systems)

\section{INTRODUCTION}

The Numerical Renormalization Group (NRG) is known as a powerful tool for the investigation of quantum impurity problems, where a quantum system with a finite number of internal degrees of freedom (the impurity) couples to an infinite system of non-interacting fermions with a continuous density of states (the bath) ${ }^{1-5}$. The NRG combines numerically exact diagonalization with the idea of the renormalization group, where progressively smaller energy scales are treated in the course of the calculation. NRG calculations are non-perturbative and - thanks to the logarithmic energy discretization are able to access arbitrarily small energies and temperatures. Besides providing thermodynamic quantities like susceptibility, entropy, and magnetization, the NRG can be used to compute dynamic observables directly on the real frequency axis.

While the NRG was originally developed by Wilson ${ }^{1}$ for the Kondo model, it was later applied to a variety of more complex impurity models with one or more fermionic baths, being able to handle, e.g., twochannel and multi-impurity physics ${ }^{6,7}$. As a recent extension, impurity models with a fermionic bath and a single bosonic mode have been treated, with the so-called Anderson-Holstein impurity model being the paradigmatic example ${ }^{8}$. Interesting applications of the NRG include its use within dynamical mean-field theory $(\text { DMFT })^{9,10}$. There, the electronic self-energy of a lattice model of correlated electrons is approximated by a local function in space, and the lattice model is mapped onto a single-impurity model supplemented by a self-consistency condition. Using DMFT-NRG, the Mott transition of the Hubbard model has been investigated in detail, both at zero and finite temperatures ${ }^{11,12}$.

The objective of this paper is an important extension of the NRG method, namely the application to quantum impurities coupled to a bosonic bath with a continuous spectral density (in contrast to the single boson mode in Ref. 8). We have recently given a short account on this development ${ }^{13}$; the purpose here is a detailed description of this novel NRG application. To be specific, most of our presentation will focus on the spin-boson model, with the Hamiltonian

$$
H=-\frac{\Delta}{2} \sigma_{x}+\frac{\epsilon}{2} \sigma_{z}+\sum_{i} \omega_{i} a_{i}^{\dagger} a_{i}+\frac{\sigma_{z}}{2} \sum_{i} \lambda_{i}\left(a_{i}+a_{i}^{\dagger}\right) .
$$

This model naturally arises in the description of quantum dissipative systems ${ }^{14,15}$ : The dynamics of the twostate system, represented by the Pauli matrices $\sigma_{x, z}$, is governed by the competition between the tunneling term $\Delta$ and the friction term $\lambda_{i}\left(a_{i}+a_{i}^{\dagger}\right)$. The $a_{i}$ constitute a bath of harmonic oscillators responsible for the damping, characterized by the bath spectral function

$$
J(\omega)=\pi \sum_{i} \lambda_{i}^{2} \delta\left(\omega-\omega_{i}\right) .
$$

Clearly, most interesting are gapless spectra, $J(\omega)>0$ for $0<\omega<\omega_{c}$, with $\omega_{c}$ being a cutoff energy. In the infrared limit, the energy dependence of $J(\omega)$ for $\omega \rightarrow 0$ determines the system's behavior, where power-laws are of particular importance. Discarding high-energy details of the spectrum, the standard parametrization is

$$
J(\omega)=2 \pi \alpha \omega_{c}^{1-s} \omega^{s}, 0<\omega<\omega_{c}, \quad s>-1 .
$$

The case $s=1$ is known as Ohmic dissipation ${ }^{14}$, where the spin-boson model has a delocalized and a localized zero-temperature phase, separated by a KosterlitzThouless transition (for the unbiased case of $\epsilon=0$ ). In the delocalized phase, realized at small dissipation strength $\alpha$, the ground state is non-degenerate and represents a (damped) tunneling particle. For large $\alpha$, the 
dissipation leads to a localization of the particle in one of the two $\sigma_{z}$ eigenstates, thus the ground state is doubly degenerate.

Bath spectra with exponents $s>1(s<1)$ are called super-Ohmic (sub-Ohmic): In the super-Ohmic case, the system is always delocalized with weak damping; the subOhmic case is more involved and will be discussed below. Besides simple power-law spectra, more complicated bath properties can arise in a number of situations, e.g., structured baths, consisting of an Ohmic part and modes sharply peaked at certain energies, have been considered recently ${ }^{16,17}$.

The spin-boson model has found applications in a wide variety of physical situations ${ }^{14,15}$ : mechanical friction, damping in electric circuits, decoherence of quantum oscillations in qubits ${ }^{18-20}$, impurity moments coupled to bulk magnetic fluctuations ${ }^{21}$, and electron transfer in biological molecules ${ }^{22,23}$.

Considering this wealth of applications, numerical methods to reliably deal with the spin-boson and related models for all temperatures are highly desirable. In the past, quantum Monte-Carlo simulations ${ }^{24}$ have been used, which, however, cannot work at arbitrarily low temperature, and cannot easily extract dynamical information on the real frequency axis. Density-matrix renormalization techniques, as employed in Ref. 25, circumvent this problem, but are not able to resolve very small energy scales. In Ref. 26, the NRG with a fermionic bath has been used, exploiting the well-established mapping of the Ohmic spin-boson model to an anisotropic Kondo model. Such a mapping is only valid for frequencies $\omega \ll \omega_{c}$ (which nevertheless encompasses most of the interesting physics), and, more seriously, is restricted to the Ohmic case ${ }^{27}$.

In a recent paper, we have presented a formulation of the NRG directly for a bosonic bath, and applied it to the spin-boson model ${ }^{13}$. While we could accurately reproduce known results for the Ohmic case, we also found that the sub-Ohmic model displays two phases as well (in agreement with Refs. 28, 29) which are separated by a non-trivial quantum phase transition. Remarkably, this phase transition was not systematically investigated before. We have studied the properties of the corresponding quantum critical points - in the phase diagram (see Fig. 1 of Ref. 13) those form a line, parametrized by the bath exponent $s$, which terminates in the Kosterlitz-Thouless transition at $s=1$. Near $s=1$ we could make contact with analytical renormalization group results, originally formulated by Kosterlitz in the context of an Ising model with long-range $1 / r^{1+s}$ interaction ${ }^{30}$.

The purpose of this paper is (i) to present in detail the implementation of the bosonic NRG method for the spinboson model, (ii) to discuss various strategies to set up the iteration scheme for the bosonic NRG, (iii) to demonstrate its feasibility by studying, in particular, the case of Ohmic damping; and compare our data with a variety of results from the literature, and (iv) to discuss possible future applications of the bosonic NRG. The physics of the sub-Ohmic spin-boson model is very rich due to the presence of a line of boundary quantum critical points; a full account of the universal critical behavior, studied using analytical and numerical methods, will be given in a forthcoming publication ${ }^{31}$.

The remainder of the paper is organized as follows: In Sec. II we introduce the formulation of the NRG for bosonic systems and highlight important differences which occur compared to the fermionic NRG. In particular, the choice of appropriate bosonic basis states, which are required to accurately describe certain strongcoupling fixed points, is discussed, with details given in Appendix A and B. Section III analyzes the NRG flow and the low-energy fixed points, the phase boundaries and issues of numerical convergence as function of the discretization parameters. In Sec. IV we turn to thermodynamic observables calculated using the bosonic NRG, such as entropy and specific heat, together with their scaling behavior. Finally, Sec. V is devoted to dynamical quantities, where we focus on the symmetrized impurity spin autocorrelation function. We close with a summary and discussion of applications and extensions of the bosonic NRG.

\section{THE BOSONIC NRG}

The bosonic NRG can be applied to a wide range of quantum impurity problems involving a bosonic bath with a continuous (and in particular gapless) spectrum. The following discussion of the technical details of this method concentrates on the spin-boson model, the first application of the bosonic NRG ${ }^{13}$. The general concepts are valid for the study of other bosonic impurity models as well.

The purpose of this section is twofold: we want to discuss in detail the technical steps of the calculations in Ref. 13. In addition, we introduce an alternative strategy to set up the NRG procedure, the so-called star-NRG, in contrast to the chain-NRG used in Ref. 13. The use of the star-NRG is related to the choice of an optimized set of basis states. As will be discussed in Secs. IIF and III D, the star-NRG allows for an efficient construction of the NRG basis which solves the problem of the boson number divergence occurring in the localized phase for sub-Ohmic damping.

Let us start with a form of the spin-boson model which is most convenient for the NRG procedure:

$$
H=H_{\mathrm{loc}}+\int_{0}^{1} \mathrm{~d} \varepsilon g(\varepsilon) a_{\varepsilon}^{\dagger} a_{\varepsilon}+\frac{\sigma_{z}}{2} \int_{0}^{1} \mathrm{~d} \varepsilon h(\varepsilon)\left(a_{\varepsilon}+a_{\varepsilon}^{\dagger}\right)
$$

with $H_{\mathrm{loc}}=-\Delta \sigma_{x} / 2+\epsilon \sigma_{z} / 2$. In this model, $g(\varepsilon)$ characterizes the dispersion of a bosonic bath in a onedimensional representation, with upper cutoff 1 for $\varepsilon$. The coupling between the spin and the bosonic bath is 


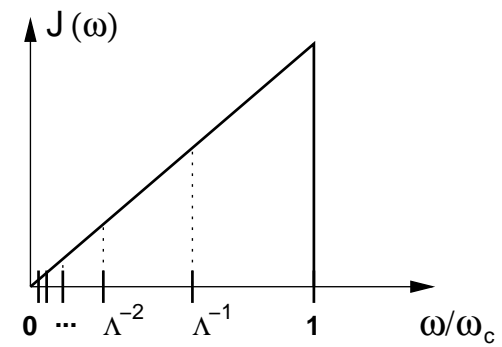

FIG. 1. Logarithmic discretization of the bath spectral function in intervals $\left[\Lambda^{-(n+1)}, \Lambda^{-n}\right](n=0,1,2, \ldots)$; typical values of the NRG discretization parameter $\Lambda$ as used in the bosonic NRG are $\Lambda=1.5 \ldots 3.0$.

given by $h(\varepsilon)$. These two energy-dependent functions are related to the spectral function $J(\omega)$ via

$$
\frac{1}{\pi} J(x)=\frac{d \varepsilon(x)}{d x} h^{2}[\varepsilon(x)] \quad\left(x \in\left[0, \omega_{c}\right]\right),
$$

where $\varepsilon(x)$ is the inverse function of $g(x), g[\varepsilon(x)]=x$. For a given $J(x)$, eq. (5) does not determine both $g$ and $h$ independently. Therefore, as shown below in eq. (10), a specific choice of $h$ is used to simplify the calculations.

\section{A. Logarithmic Discretization}

The NRG procedure starts by dividing the interval $[0,1]$ into intervals $\left[\Lambda^{-(n+1)}, \Lambda^{-n}\right](n=0,1,2, \ldots$, see Fig. 1). The width of each interval is

$$
d_{n}=\Lambda^{-n}\left(1-\Lambda^{-1}\right)
$$

Within each interval we introduce a complete set of orthonormal functions:

$$
\psi_{n p}(\varepsilon)=\left\{\begin{array}{ll}
\frac{1}{\sqrt{d_{n}}} e^{i \omega_{n} p \varepsilon} & \text { for } \Lambda^{-(n+1)}<\varepsilon<\Lambda^{-n} \\
0 & \text { outside this interval }
\end{array},\right.
$$

$\left(p=0, \pm 1, \pm 2, \ldots\right.$ and $\left.\omega_{n}=2 \pi / d_{n}\right)$. The operators $a_{\varepsilon}^{(\dagger)}$ appearing in the Hamiltonian (4) can be represented in this basis:

$$
\begin{aligned}
& a_{\varepsilon}=\sum_{n p} a_{n p} \psi_{n p}(\varepsilon) \\
& a_{\varepsilon}^{\dagger}=\sum_{n p} a_{n p}^{\dagger} \psi_{n p}^{*}(\varepsilon) .
\end{aligned}
$$

We then choose the function $h(\varepsilon)$ to be a constant $h_{n}$ in each intervall of the logarithmic discretization:

$$
h(\varepsilon)=h_{n}=\left[\frac{1}{\Lambda^{-n}-\Lambda^{-(n+1)}} \int_{\Lambda^{-(n+1)} \omega_{c}}^{\Lambda^{-n} \omega_{c}} \frac{1}{\pi} J(\omega) \mathrm{d} \omega\right]^{1 / 2}
$$

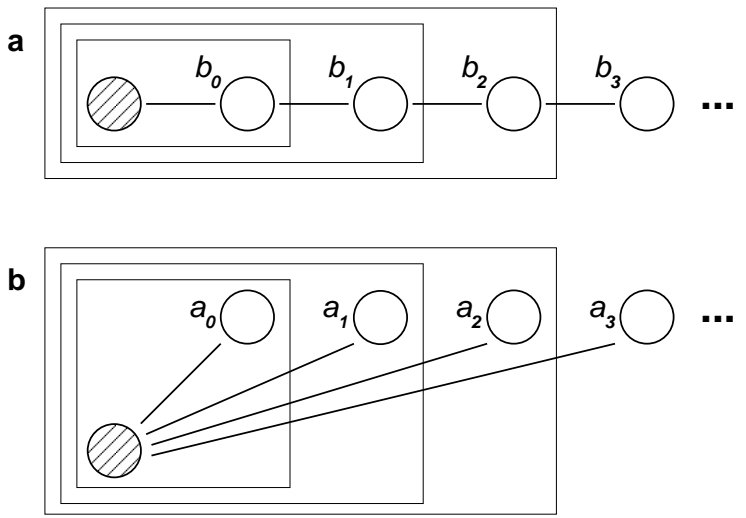

FIG. 2. (a) Structure of the spin-boson model corresponding to eq. (14) as used for the chain-NRG and (b) to eq. (11) as used for the star-NRG; the boxes indicate the iterative diagonalization scheme for both cases.

for $\varepsilon \in\left[\Lambda^{-(n+1)}, \Lambda^{-n}\right]$. With this choice, the impurity (the spin-operator $\sigma_{z}$ ) couples to the $p=0$ component of the bosonic operators $a_{n p}$ and $a_{n p}^{\dagger}$ only (the same strategy has been used in the case of a fermionic bath with non-constant density of states, see Ref. 32).

The next step is to write the Hamiltonian (4) in the basis $a_{n p}$ and $a_{n p}^{\dagger}$; the $p \neq 0$ components of these operators are still present through their coupling to the $p=0$ components in the free bath term. The main approximation of the bosonic NRG at this point is to drop this coupling, in close analogy to the fermionic case (see Refs. 1, 2). This approximation becomes exact in the limit $\Lambda \rightarrow 1$. Nevertheless, a careful check of its validity is necessary and will be discussed in Sec. III B.

With the $p \neq 0$ components completely decoupled from the impurity, we drop the $p=0$ index in the operators $a_{n p=0}$ and $a_{n p=0}^{\dagger}$ and arrive at a Hamiltonian of the form:

$$
H_{\mathrm{s}}=H_{\mathrm{loc}}+\sum_{n=0}^{\infty} \xi_{n} a_{n}^{\dagger} a_{n}+\frac{\sigma_{z}}{2 \sqrt{\pi}} \sum_{n=0}^{\infty} \gamma_{n}\left(a_{n}+a_{n}^{\dagger}\right)
$$

with

$$
\left.\xi_{n}=\gamma_{n}^{-2} \int_{\Lambda^{-(n+1)} \omega_{c}}^{\Lambda^{-n} \omega_{c}} \mathrm{~d} x J(x) x, \quad \gamma_{n}^{2}=\int_{\Lambda^{-(n+1)} \omega_{c}}^{\Lambda^{-n} \omega_{c}} \mathrm{~d} x J\right) .
$$

The label $H_{\mathrm{s}}$ is introduced to distinguish this "star"Hamiltonian from the "chain"-Hamiltonian $H_{\mathrm{c}}$ (see eq. (14) below). The $\xi_{n}$ and the $\gamma_{n}$ can be easily evaluated for a bath spectral function of the form given in eq. (3):

$$
\begin{aligned}
\xi_{n} & =\frac{s+1}{s+2} \frac{1-\Lambda^{-(s+2)}}{1-\Lambda^{-(s+1)}} \omega_{c} \Lambda^{-n} \\
\gamma_{n}^{2} & =\frac{2 \pi \alpha}{s+1} \omega_{c}^{2}\left(1-\Lambda^{-(s+1)}\right) \Lambda^{-n(s+1)} .
\end{aligned}
$$

The structure of this Hamiltonian is sketched in Fig. 2b: the impurity spin couples linearly to all the 
bosonic degrees of freedom $a_{n}$, in a very similar way as in the original Hamiltonian (1). The bath spectral function for model (11) is discrete, consisting of $\delta$-peaks at energies $\xi_{n}$ with weight $\propto \gamma_{n}^{2}$. Each bosonic degree of freedom of this star-Hamiltonian is a representative of the continuous spectrum of bosonic degrees of freedom in the intervals $\left[\Lambda^{-(n+1)}, \Lambda^{-n}\right]$.

\section{B. Chain-NRG vs. Star-NRG}

Starting from the model (11), there are two possible ways to set up a numerical renormalization group procedure. The first one (which we call "chain"-NRG in the following) uses the transformation of the starHamiltonian (11) to a semi-infinite chain:

$$
\begin{aligned}
H_{\mathrm{c}} & =H_{\mathrm{loc}}+\sqrt{\frac{\eta_{0}}{\pi}} \frac{\sigma_{z}}{2}\left(b_{0}+b_{0}^{\dagger}\right) \\
& +\sum_{n=0}^{\infty}\left[\epsilon_{n} b_{n}^{\dagger} b_{n}+t_{n}\left(b_{n}^{\dagger} b_{n+1}+b_{n+1}^{\dagger} b_{n}\right)\right],
\end{aligned}
$$

with $\eta_{0}=\int \mathrm{d} x J(x)$. The spin now couples to the first site of the bosonic chain only (see Fig. 2a) and the remaining part of the chain is characterized by on-site energies $\epsilon_{n}$ and hopping parameters $t_{n}$, in analogy to the fermionic NRG. The parameters $\epsilon_{n}$ and $t_{n}$ can be calculated numerically from a given spectral function $J(\omega)$, as discussed in detail in Appendix A.

Such a mapping from a star-Hamiltonian on a semiinfinite chain form is exact. It has been used in all applications of the fermionic NRG since the original work of Wilson ${ }^{1}$. Its generalization to the bosonic NRG is straightforward and has been employed in Ref. 13 (see also Ref. 33).

The structure of the Hamiltonian (14) is sketched in Fig. 2a. The boxes indicate the NRG strategy used in this case: in the first step, a cluster containing the impurity plus the first bath site is diagonalized. In each subsequent step, the cluster is enlarged by one additional site and the new cluster is diagonalized using the information obtained in the previous step.

The second possibility (which we call "star"-NRG in the following) is to use the Hamiltonian (11) directly for the iterative diagonalization. The general idea is sketched in Fig. 2b: again, the first step of the renormalization group procedure involves the diagonalization of a cluster containing the impurity plus the first bath site. The following renormalization group steps, however, are completely different to the chain-NRG as each new bosonic site does not couple to the previously added site but to the impurity instead.

The suggestion to use such a star-NRG for the investigation of bosonic impurity models, such as the spin-boson model, raises a couple of questions:

(1) Does the star-NRG work at all?
(2) Is the star-NRG of any advantage as compared to the chain-NRG (apart from the simplification that we do not have to calculate the $\epsilon_{n}$ and $t_{n}$ of eq. $(14))$ ?

(3) Why has such a star-NRG not been used in the fermionic case?

The answers to questions No. (1) and (2) will be given further below. Let us first discuss question No. (3) in more detail. A fermionic star-NRG for, say, the Kondo model would start from a Hamiltonian similar to eq. (11). The important difference in the fermionic case is that the logarithmic discretization has to be performed for both positive and negative frequencies. As a consequence, there are two sets of bath operators in the starHamiltonian, one for positive and one for negative frequencies:

$$
\sum_{\sigma, n=0}^{\infty} \xi_{n}^{+} f_{\sigma, n+}^{\dagger} f_{\sigma, n+}+\sum_{\sigma, n=0}^{\infty} \xi_{n}^{-} f_{\sigma, n-}^{\dagger} f_{\sigma, n-} .
$$

For a hybridization function close to particle-hole symmetry we have $\xi_{n}^{+} \approx \xi_{n}^{-}$. This means that at each renormalization group step one has to add two fermionic sites (the alternative to add $f_{\sigma, n+}^{(\dagger)}$ first and then $f_{\sigma, n-}^{(\dagger)}$, or vice versa, suffers from violating particle-hole symmetry, if present). The Hilbert space therefore increases by a factor of 16 in each step. It is much more convenient to first map the star-Hamiltonian to a chain form similar to eq. (14). In this form, only one site has to be added in each renormalization group step.

Whether such a fermionic star-NRG is of any advantage is not clear. It might be useful for extreme asymmetric cases, but for the cases which are usually of interest the chain-NRG already works very well and is much easier to implement.

Coming back to the bosonic NRG, there does not seem to be an a priori preference for either star- or chain-NRG because the structure of the bosonic bath is extremely asymmetric from the outset (restricted to positive frequencies only). To address the possible advantages of the star-NRG, we first have to give more details of how the bosonic NRG is implemented (for both star- and chainNRG).

\section{Iterative Diagonalization and Choice of Bosonic Basis States}

The star-Hamiltonian $H=H_{\mathrm{s}}$ (11) and the chainHamiltonian $H=H_{\mathrm{c}}$ (14) can be written as a series of Hamiltonians $H_{N}(N \geq 0)$ equal to $H$ in the limit $N \rightarrow \infty:$

$$
H=\lim _{N \rightarrow \infty} \Lambda^{-N} H_{N}
$$

The $H_{N}$ for the star-Hamiltonian are given by 
$H_{N, \mathrm{~s}}=\Lambda^{N}\left[H_{\mathrm{loc}}+\sum_{n=0}^{N} \xi_{n} a_{n}^{\dagger} a_{n}+\frac{\sigma_{z}}{2 \sqrt{\pi}} \sum_{n=0}^{N} \gamma_{n}\left(a_{n}+a_{n}^{\dagger}\right)\right]$,

and for the chain-Hamiltonian by

$$
\begin{aligned}
H_{N, \mathrm{c}} & =\Lambda^{N}\left[H_{\mathrm{loc}}+\sqrt{\frac{\eta_{0}}{\pi}} \frac{\sigma_{z}}{2}\left(b_{0}+b_{0}^{\dagger}\right)\right. \\
& \left.+\sum_{n=0}^{N} \epsilon_{n} b_{n}^{\dagger} b_{n}+\sum_{n=0}^{N-1} t_{n}\left(b_{n}^{\dagger} b_{n+1}+b_{n+1}^{\dagger} b_{n}\right)\right] .
\end{aligned}
$$

In this notation, both $H_{0, \mathrm{~s}}$ and $H_{0, \mathrm{c}}$ correspond to a twosite Hamiltonian with only the first site of the star or chain coupled to the spin.

Two successive Hamiltonians are related by the following renormalization group transformations:

$$
\begin{aligned}
& H_{N+1, \mathrm{~s}}=\Lambda H_{N, \mathrm{~s}} \\
+ & \Lambda^{N+1}\left[\xi_{N+1} a_{N+1}^{\dagger} a_{N+1}+\frac{\sigma_{z}}{2 \sqrt{\pi}} \gamma_{N+1}\left(a_{N+1}+a_{N+1}^{\dagger}\right)\right],
\end{aligned}
$$

and

$$
\begin{aligned}
& H_{N+1, \mathrm{c}}=\Lambda H_{N, \mathrm{c}} \\
+ & \Lambda^{N+1}\left[\epsilon_{N+1} b_{N+1}^{\dagger} b_{N+1}+t_{N}\left(b_{N}^{\dagger} b_{N+1}+b_{N+1}^{\dagger} b_{N}\right)\right] .
\end{aligned}
$$

The factor $\Lambda^{N}$ in eqs. (17) and (18) enables the direct comparison of the low-frequency spectra of subsequent Hamiltonians and, in particular, the discussion of fixed points as in Sec. III. In contrast to the fermionic case, the factor is $\Lambda^{N}$ instead of $\Lambda^{N / 2}$ because the energies $\xi_{n}$ in the star-Hamiltonian and the $\epsilon_{n}$ and $t_{n}$ in the chainHamiltonian are falling off as $\Lambda^{-n}$, instead of the $t_{n} \propto$ $\Lambda^{-n / 2}$ in the fermionic case. (This implies that a bosonic NRG calculation with discretization parameter $\Lambda$ and a particle-hole symmetric fermionic one with $\Lambda^{2}$ will have comparable energy resolution.) Note that, in the subOhmic spin-boson case, the $\gamma_{n}$ are falling off slower than $\Lambda^{-n}$. Nevertheless, the factor $\Lambda^{-n}$ is the appropriate one for the low-energy spectra as shown in Sec. IIE.

The sequences of Hamiltonians (17) and (18) are solved by iterative diagonalization. In the first step, the $H_{0}$ are diagonalized in a basis formed by the product states of $\sigma_{z}$-eigenstates $|\sigma\rangle$ and a suitable basis for the first bath site (we will describe below what we mean with 'suitable basis'). We have to introduce a cutoff $N_{\mathrm{b} 0}$ already for this basis, but this is usually not a serious restriction as we can use fairly large values of $N_{\mathrm{b} 0} \approx 500$ (in contrast to the much lower values of $N_{\mathrm{b}}$ for the following iterations).

Given the eigenstates $|r\rangle_{N}$ of $H_{N}$

$$
H_{N}|r\rangle_{N}=E_{N}(r)|r\rangle_{N} \quad, \quad r=1, \ldots N_{\mathrm{s}},
$$

with $N_{\mathrm{s}}$ the dimension of $H_{N}$, we can construct a basis of $H_{N+1}$ :

$$
|r ; s\rangle_{N+1}=|r\rangle_{N} \otimes|s(N+1)\rangle,
$$

with $|s(N+1)\rangle$ a suitable basis for the added site. In setting up the basis $|s(N+1)\rangle$ we are faced with two problems not present in the fermionic case:

(1) The numerical approach restricts the number of basis states one can take into account to a maximum number $N_{\mathrm{b}} \approx 10-14$. The validity of this approximation has to be checked carefully.

(2) A criterion for a suitable selection of $N_{\mathrm{b}}$ basis states out of the infinitely many states of the added site has to be found.

A general criterion for an "optimal" basis (for a given $N_{\mathrm{b}}$ ) can be formulated as following: find a set of $N_{\mathrm{b}}$ boson states $|s(N+1)\rangle$ which give the best description of the lowest-lying many-particle states of $H_{N+1}$ (see also Ref. 34). In a variational sense, this corresponds to finding states $|s(N+1)\rangle$ which give the lowest many-particle energies for a whole set of energy levels (see also Fig. 4 below). This is certainly not a rigorous statement and we have not yet developed a general algorithm to set up such an optimal basis. Instead we select one of the two sets of basis states optimized for the two stable fixed points of the spin-boson model: the $N_{\mathrm{b}}$ eigenstates of $b_{N+1}^{\dagger} b_{N+1}$ (or $a_{N+1}^{\dagger} a_{N+1}$ ) with lowest eigenvalues as an optimal basis for the delocalized fixed point (Sec. IID) and displaced oscillators as optimal basis for the localized fixed point (Sec. IIE).

Before continuing let us point out that are no symmetries in the Hamiltonians $H_{N, \mathrm{~s}}$ and $H_{N, \mathrm{c}}$ (at least for the interesting case of finite $\alpha$ and $\Delta$ ). This is in contrast to the fermionic case ${ }^{1,2}$, where we can use, for example, the total spin and particle number as quantum numbers to significantly reduce the size of the Hamiltonian matrices (which would be of size $\left(4 N_{\mathrm{s}}\right)^{2}$ in the absence of symmetries). Consequently, in the bosonic NRG for the spinboson model there is only one matrix of size $\left(N_{\mathrm{b}} N_{\mathrm{s}}\right)^{2}$ to be diagonalized in each renormalization group step. This results in a much simpler structure of the NRG program, but limits the values of $N_{\mathrm{s}}$ to $100-200$.

\section{Optimal Basis for the Delocalized Fixed Point}

Let us start from the $\alpha=0$ limit of the spin-boson model in which two-level system and bosonic degrees of freedom are completely decoupled; for finite $\Delta$, the spin oscillations are undamped and the system is in the delocalized phase from the outset.

The Hamiltonian in the original formulation (1) then takes the form:

$$
H=-\frac{\Delta}{2} \sigma_{x}+\sum_{i} \omega_{i} a_{i}^{\dagger} a_{i} .
$$


For simplicity, the bias $\epsilon$ is set to zero. The starHamiltonian in the $\alpha=0$ limit has the same structure:

$$
H_{\mathrm{s}}=-\frac{\Delta}{2} \sigma_{x}+\sum_{n} \xi_{n} a_{n}^{\dagger} a_{n}
$$

From this structure, it is clear that the $N_{\mathrm{b}}$ eigenstates of $a_{N+1}^{\dagger} a_{N+1}$ with lowest eigenvalues form the optimal basis:

$$
|s(N+1)\rangle=\left\{\left|n_{N+1}\right\rangle\right\}
$$

with

$$
a_{N+1}^{\dagger} a_{N+1}\left|n_{N+1}\right\rangle=n\left|n_{N+1}\right\rangle, \quad n=0,1, \ldots N_{\mathrm{b}}-1 .
$$

The reason is simply that here the many-particle energies are given by the sum of the single-particle energies $\xi_{n}$.

The situation is similar in the chain-NRG, where the $\alpha=0$ limit reads:

$$
H_{\mathrm{c}}=-\frac{\Delta}{2} \sigma_{x}+\sum_{n=0}^{\infty}\left[\epsilon_{n} b_{n}^{\dagger} b_{n}+t_{n}\left(b_{n}^{\dagger} b_{n+1}+b_{n+1}^{\dagger} b_{n}\right)\right] \text {. }
$$

Here we choose for the basis $|s(N+1)\rangle$ the states $\left\{\left|n_{N+1}\right\rangle\right\}$ with

$$
b_{N+1}^{\dagger} b_{N+1}\left|n_{N+1}\right\rangle=n\left|n_{N+1}\right\rangle, \quad n=0,1, \ldots N_{\mathrm{b}}-1 .
$$

The difference to the basis for the star-Hamiltonian is that the $\left|n_{N+1}\right\rangle$ are not eigenstates of the full bosonic part in eq. (27). But in contrast to the case of $\alpha>0$, the Hamiltonian (27) conserves the total number of bosons; the many-particle states with the lowest energies are then given by those states which are constructed from the single-particle states with the smallest boson numbers, independent of whether a diagonal basis is chosen or not.

In our previous implementation of the bosonic $\mathrm{NRG}^{13}$ we used the basis (28). This is a suitable choice only if the many particle states of $H_{N+1}$ with lowest energies are indeed constructed from states with small boson number - in other words, if the average values of the boson numbers $\left\langle b_{N+1}^{\dagger} b_{N+1}\right\rangle$ are small. This is the case when the system is close to the delocalized and the quantum critical fixed points. However, the boson number diverges when the system flows to the localized fixed point for $s<1$ as discussed below.

\section{E. Optimal Basis for the Localized Fixed Point (Displaced Oscillators)}

Here we consider the spin-boson model with zero tunneling amplitude, $\Delta=0$. In this case, oscillations between $|\uparrow\rangle$ and $|\downarrow\rangle$ are absent and the system is in the localized phase from the outset.
The Hamiltonian in the original formulation (1) then takes the form:

$$
H=\sum_{i} \omega_{i} a_{i}^{\dagger} a_{i}+\frac{\sigma_{z}}{2} \sum_{i} \lambda_{i}\left(a_{i}+a_{i}^{\dagger}\right) .
$$

For simplicity, the bias $\epsilon$ is set to zero. As the bath degrees of freedom now couple to a static spin, the Hamiltonian can be decomposed in two sectors $H_{\uparrow}$ for $\sigma_{z}=+1$ and $H_{\downarrow}$ for $\sigma_{z}=-1$ :

$$
H_{\uparrow}=\sum_{i} H_{i \uparrow}, \quad H_{i \uparrow}=\omega_{i} a_{i}^{\dagger} a_{i}+\frac{\lambda_{i}}{2}\left(a_{i}+a_{i}^{\dagger}\right),
$$

( $H_{\downarrow}$ accordingly). In each sector, we now have independent bosonic degrees of freedom which can be written as:

$$
H_{i \uparrow}=\omega_{i} \bar{a}_{i}^{\dagger} \bar{a}_{i}
$$

(dropping a constant term) with

$$
\bar{a}_{i}=a_{i}+\theta_{i} \quad, \quad \theta_{i}=\frac{\lambda_{i}}{2 \omega_{i}} .
$$

The quantities $\theta_{i}$ can be viewed as an effective (dimensionless) coupling between impurity and bath mode $i$. Apparently, this transformation corresponds to a displacement of the oscillators $a_{i}$ by the value $+\theta_{i}$ for the $\uparrow$-sector and $-\theta_{i}$ for the $\downarrow$-sector. The displacements do not change the energies $\omega_{i}$. This means that the whole many-particle spectrum of the bosonic bath is identical to the one for the uncoupled bath except for the additional two-fold degeneracy corresponding to the two sectors $\uparrow$ and $\downarrow$.

Note that for the original spin-boson model (1) the $\omega_{i}$ and $\lambda_{i}$ are not specified independently, only the bath spectral function $J(\omega)$ is given; therefore we cannot give explicit expressions for the $\theta_{i}$ for eq. (1).

The star-Hamiltonian eq. (11) for $\Delta=0$ (and $\epsilon=0$ ) takes a form similar to eq. (29). Again we have two sectors with

$$
H_{\mathrm{s} \uparrow}=\sum_{n=0}^{\infty} \xi_{n} a_{n}^{\dagger} a_{n}+\frac{1}{2 \sqrt{\pi}} \sum_{n=0}^{\infty} \gamma_{n}\left(a_{n}+a_{n}^{\dagger}\right),
$$

( $H_{\mathrm{s} \downarrow}$ accordingly). Using the same reasoning as above, we can now write

$$
H_{\mathrm{s} \uparrow}=\sum_{n=0}^{\infty} \xi_{n} \bar{a}_{n}^{\dagger} \bar{a}_{n}
$$

with

$$
\bar{a}_{n}=a_{n}+\theta_{n} \quad, \quad \theta_{n}=\frac{\gamma_{n}}{2 \sqrt{\pi} \xi_{n}}
$$

The values of $\gamma_{n}$ and $\xi_{n}$ are given in eq. (13) so we obtain

$$
\theta_{n} \propto \Lambda^{n(1-s) / 2} \propto \xi_{n}^{(s-1) / 2}
$$


Written in terms of energy $\omega$ we therefore have:

$$
\theta(\omega) \propto \omega^{(s-1) / 2} .
$$

This result is rather interesting: for sub-Ohmic baths, $s<1$, the shift $\theta_{n}$ grows exponentially with $n$. However, in the super-Ohmic case the shift goes to zero in the lowenergy limit $(n \rightarrow \infty)$, and it is energy-independent for the Ohmic case. Technically, the coupling to the impurity can be viewed as a relevant (irrelevant) perturbation of the discretized spin-boson model for $s<1(s>1)$ and as a marginal perturbation in the Ohmic case. Thus, in the Ohmic and super-Ohmic case the effective coupling $\theta(\omega)$ does not diverge as $\omega \rightarrow 0$ even in the extreme localized case of $\Delta=0$. Therefore, numerical problems associated with a diverging effective coupling are only expected in the sub-Ohmic case.

Coming back to the iterative numerical diagonalization of the star-Hamiltonian, it is now clear that a simple basis as in (25) can be far from the optimal choice. If we stay in the original basis constructed from the lowest eigenstates of $a_{n}^{\dagger} a_{n}$, we need more and more basis states to describe the lowest eigenstates of the displaced oscillators.

On the other hand, it is clear how to construct the optimal basis for $|s(N+1)\rangle$ at least for the $\Delta=0$ case. For the sectors $\uparrow / \downarrow$ we simply take oscillator states with displacements $+\theta_{N+1} /-\theta_{N+1}$. As we need a single basis for both sectors, these states have to be orthogonalized first; this will be discussed in more detail in Appendix B.

The displaced oscillator states can also be used to diagonalize the chain-Hamiltonian (14) for $\Delta=0$. For a given iteration number $N$, the $H_{N, \mathrm{c}}$ for the $\uparrow$-sector reads:

$$
\begin{aligned}
H_{N, \mathrm{c} \uparrow} & =\Lambda^{N}\left[\frac{1}{2} \sqrt{\frac{\eta_{0}}{\pi}}\left(b_{0}+b_{0}^{\dagger}\right)\right. \\
& \left.+\sum_{n=0}^{N} \epsilon_{n} b_{n}^{\dagger} b_{n}+\sum_{n=0}^{N-1} t_{n}\left(b_{n}^{\dagger} b_{n+1}+b_{n+1}^{\dagger} b_{n}\right)\right] .
\end{aligned}
$$

Introducing displaced oscillators

$$
\bar{b}_{n}=b_{n}+\theta_{n}(N),
$$

we again have a diagonal form

$$
H_{N, \mathrm{c} \uparrow}=\Lambda^{N} \sum_{n=0}^{N} \epsilon_{n} \bar{b}_{n}^{\dagger} \bar{b}_{n} .
$$

The displacements $\theta_{n}(N)$ can be calculated numerically for any given set of $\left\{\epsilon_{n}\right\}$ and $\left\{t_{n}\right\}$. For fixed $N$ they show the same qualitative behavior as the $\theta_{n}$ for the starHamiltonian:

$$
\left|\theta_{n}(N)\right| \propto \Lambda^{n(1-s) / 2} .
$$

It turns out, however, that the $\theta_{n}(N)$ depend on both $n$ and $N$ with significant deviations from the exponential form for $n$ close to $N$. This has important consequences for the use of displaced oscillators as basis states in the chain-NRG. Let us assume that we used $\pm \theta_{N}(N)$ to construct the basis for $H_{N, \mathrm{c}}$. Adding the site $N+1$ introduces a significant change in the displacement $\theta_{N}(N) \rightarrow \theta_{N}(N+1)$. One possible solution to this problem is to anticipate the coupling to the site $N+1$ by adding a static displacement term, which is subtracted again in the next step. Such an approach gives correct results for the chain-NRG when we set $\Delta=0$. We did not, however, succeed in implementing the displaced oscillator idea for the general case of finite $\Delta$ in the chainNRG. So far, this strategy only works for the star-NRG as described in the following subsection.

\section{F. General Strategy of the Bosonic NRG}

In the preceding subsections we have described various options of how to set up the bosonic NRG. We have introduced both a star- and a chain-representation of the spinboson model and we discussed two possibilities for choosing a basis for the added site: eigenstates of $b_{N+1}^{\dagger} b_{N+1}$ (or $a_{N+1}^{\dagger} a_{N+1}$ ) as in Sec. IID or displaced oscillators as in Sec. II E.

Now we want to discuss how we actually proceed with the bosonic NRG: how do we decide between the different options described above?

As a starting point we choose the chain-NRG using eigenstates of $b_{N+1}^{\dagger} b_{N+1}$ (the basis denoted by $\left.\left|n_{N+1}\right\rangle\right)$ as the simplest possible basis. This approach has been used for all the results shown in Ref. 13. From the discussion in Secs. II D and II E we anticipate that this choice of the basis is reasonable for

- all parameters in the super-Ohmic case,

- the Ohmic case provided the coupling $\alpha$ is not too large,

- and the sub-Ohmic case provided the system is close to the delocalized fixed point.

On the other hand, it is clear that there will be problems when the system flows to the localized fixed point in the sub-Ohmic case. The situation in the crossover regions and close to the quantum critical points needs to be checked numerically: it turns out that the critical fixed points for all $0<s<1$ can be reached using the $\left|n_{N+1}\right\rangle$ basis.

There is a simple criterion to decide when the basis $\left|n_{N+1}\right\rangle$ is sufficient. Consider the expectation value $n_{N+1}\left(N_{\mathrm{b}}\right)=\left\langle b_{N+1}^{\dagger} b_{N+1}\right\rangle$ for the cluster after adding the site $N+1$, calculated for a temperature of the order of the level spacing at this NRG step. This quantity can be obtained numerically up to values of $N_{\mathrm{b}} \approx 14$. If the lowest eigenvalues of $b_{N+1}^{\dagger} b_{N+1}$ are a good choice for describing the lowest eigenstates of $H_{N+1}$, then $n_{N+1}\left(N_{\mathrm{b}}\right)$ should be small and rapidly saturate with increasing $N_{\mathrm{b}}$. If, on the other hand, we identify that $n_{N+1}\left(N_{\mathrm{b}}\right)$ does not 


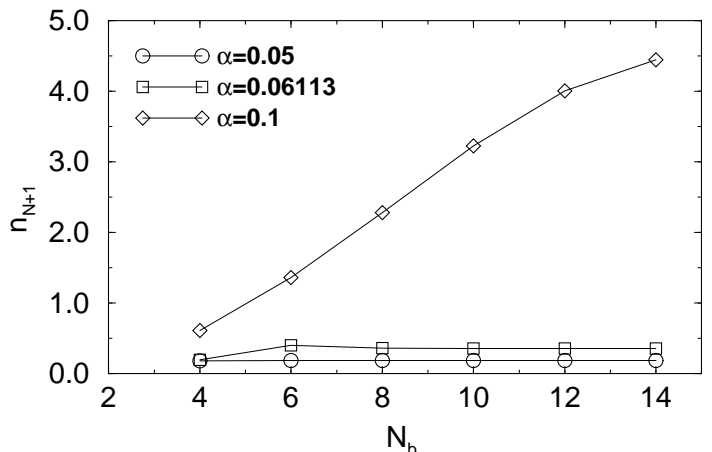

FIG. 3. Dependence of the expectation value $n_{N+1}=\left\langle b_{N+1}^{\dagger} b_{N+1}\right\rangle$ on the number of basis states $N_{\mathrm{b}}$ for a chain-NRG calculation. The values of $n_{N+1}\left(N_{\mathrm{b}}\right)$ quickly saturate for $\alpha=0.05<\alpha_{\mathrm{c}}$ and $\alpha_{\mathrm{c}}=0.06113$ whereas no saturation is observed for $\alpha=0.1>\alpha_{\mathrm{c}}$. (Parameters are $\left.s=0.6, N=20, \Lambda=2.0, N_{\mathrm{s}}=60, \Delta=0.01\right)$.

saturate but increases with $N_{\mathrm{b}}$, then we certainly have to abandon the basis $\left|n_{N+1}\right\rangle$ and use a different 'optimized' basis.

This behavior is shown in Fig. 3 where we show results from the chain-NRG for a sub-Ohmic bath $(s=0.6)$, $\Delta=0.01$ and three values of $\alpha$ in the vicinity of the quantum phase transition. For $\alpha<\alpha_{\mathrm{c}}$ and $\alpha=\alpha_{\mathrm{c}}$ we indeed find a rapid saturation of $n_{N+1}\left(N_{\mathrm{b}}\right)$ whereas no saturation (at least up to $N_{\mathrm{b}}=14$ ) is observed for $\alpha>$ $\alpha_{\mathrm{c}}$.

The behavior of $n_{N+1}\left(N_{\mathrm{b}}\right)$ for $\alpha>\alpha_{\mathrm{c}}$ can be easily understood from the discussion of Sec. II E: as the system is flowing to the localized fixed point corresponding to the effective $\Delta$ approaching zero, we have to use properly displaced oscillators as a basis. The increase of $n_{N+1}\left(N_{\mathrm{b}}\right)$ just means that we need more and more states in the undisplaced basis to describe the lowest eigenstates of $H_{N+1}$.

In this case, the use of displaced oscillators as introduced in Sec. IIE is much more appropriate. Note, however, that the shifts $\theta_{n}$ eq. (35) can only be defined from the outset for the $\Delta=0$ case. For any finite $\Delta$, the system evolves according to the iterative diagonalization. If the system turns out to flow to the localized fixed point, we have to use effective displacements $\theta_{n}$ to set up the basis. These displacements have to be extracted numerically from the renormalization group calculation and are different (for finite $\Delta$ ) from the $\theta_{n}$ given in eq. (35).

Figure 4 describes the general strategy to determine the optimal values of the displacements. The low-energy spectrum of $H_{N+1}$ is calculated for a whole set of $\theta$ values. According to the discussion in Sec. IIC, we identify the optimal $\theta$ as the one which gives the lowest eigenenergies in $H_{N+1}$. This value is indicated by the vertical line in Fig. 4, which shows results for the subOhmic case and parameters close to the localized fixed point. There is a plateau in the energy levels close to the optimal value which means that a slight variation of the $\theta$ affects the lowest energies only very weakly. Note that

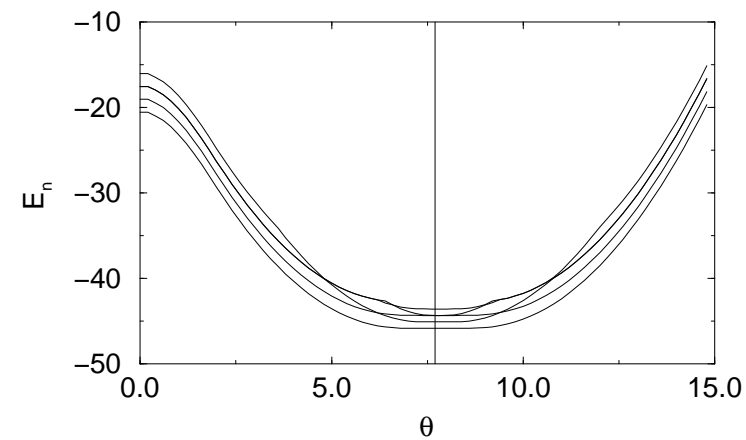

FIG. 4. Dependence of the energies of the lowest eigenstates of $H_{N+1}$ on the displacement $\theta$ used for constructing the basis for the new degree of freedom added in each iteration step. All levels shown here have their minimum at the same value $\theta=\theta^{*}$ (indicated by the vertical line) which is the optimal value for setting up the basis. The parameters for this calculation are: $s=0.2, \Delta=1.0, \alpha=0.25>\alpha_{\mathrm{c}}$.

$E_{n}(\theta)=E_{n}(-\theta)$, therefore a maximum at $\theta=0$. The corresponding figure for parameters close to the delocalized fixed point (not shown here) gives a minimum of the many-particle levels at $\theta=0$. For further details of this procedure, see Appendix B.

The data of Fig. 4 are calculated using the star-NRG formulation. Although a similar figure can be generated using the chain-NRG, we are facing the (so far unsolved) problem discussed in II E: adding a new site changes the optimal displacements for the previous iterations. For this reason, all the results in this paper using a basis of displaced oscillators are calculated within a star-NRG representation.

\section{G. Diagonalization and Truncation}

To conclude Sec. II, let us briefly discuss the remaining technical steps necessary to complete the iterative diagonalization. For a given basis, we first set up the Hamiltonian matrices

$$
H_{N+1}\left(r s, r^{\prime} s^{\prime}\right)={ }_{N+1}\left\langle r ; s\left|H_{N+1}\right| r^{\prime} ; s^{\prime}\right\rangle_{N+1} .
$$

For both chain- and star-formulation of the NRG, the matrices can be written as a sum of three parts:

$$
H_{N+1}\left(r s, r^{\prime} s^{\prime}\right)=H_{N+1}^{(1)}+H_{N+1}^{(2)}+H_{N+1}^{(3)},
$$

with

$$
\begin{aligned}
H_{N+1}^{(1)}\left(r s, r^{\prime} s^{\prime}\right) & =\Lambda_{N+1}\left\langle r ; s\left|H_{N}\right| r^{\prime} ; s^{\prime}\right\rangle_{N+1} \\
& =\Lambda E_{N}(r) \delta_{r r^{\prime}} \delta_{s s^{\prime}}
\end{aligned}
$$

for both chain- and star-formulation and

$$
\begin{aligned}
& H_{N+1, \mathrm{~s}}^{(2)}\left(r s, r^{\prime} s^{\prime}\right) \\
& =\Lambda^{N+1} \xi_{N+1}{ }_{N+1}\left\langle r ; s\left|a_{N+1}^{\dagger} a_{N+1}\right| r^{\prime} ; s^{\prime}\right\rangle_{N+1} \\
& =\Lambda^{N+1} \xi_{N+1} \delta_{r r^{\prime}}\left\langle s(N+1)\left|a_{N+1}^{\dagger} a_{N+1}\right| s^{\prime}(N+1)\right\rangle,
\end{aligned}
$$


(with operators $a$ replaced by $b$ for $H_{N+1, \mathrm{c}}^{(2)}$ ).

The third term takes the following form for the starNRG

$$
\begin{aligned}
& H_{N+1, \mathrm{~s}}^{(3)}\left(r s, r^{\prime} s^{\prime}\right) \\
= & \Lambda^{N+1} \frac{\gamma_{N+1}}{2 \sqrt{\pi}}{ }_{N+1}\left\langle r ; s\left|\sigma_{z}\left(a_{N+1}+a_{N+1}^{\dagger}\right)\right| r^{\prime} ; s^{\prime}\right\rangle_{N+1} \\
= & \Lambda^{N+1} \frac{\gamma_{N+1}}{2 \sqrt{\pi}}{ }_{N}\left\langle r\left|\sigma_{z}\right| r^{\prime}\right\rangle_{N} \\
& \times\left\langle s(N+1)\left|a_{N+1}+a_{N+1}^{\dagger}\right| s^{\prime}(N+1)\right\rangle
\end{aligned}
$$

and for the chain-NRG

$$
\begin{aligned}
H_{N+1, \mathrm{c}}^{(3)}\left(r s, r^{\prime} s^{\prime}\right) \\
=\Lambda^{N+1} t_{N}{ }_{N+1}\langle r ; s| b_{N}^{\dagger} b_{N+1}+\text { h.c. }\left|r^{\prime} ; s^{\prime}\right\rangle_{N+1} \\
=\Lambda^{N+1} t_{N N}\left\langle r\left|b_{N}^{\dagger}\right| r^{\prime}\right\rangle_{N}\left\langle s(N+1)\left|b_{N+1}\right| s^{\prime}(N+1)\right\rangle \\
\quad+\text { h.c. }
\end{aligned}
$$

All matrix elements of the form $\left\langle s(N+1)|\ldots| s^{\prime}(N+1)\right\rangle$ can be further simplified once the basis $|s(N+1)\rangle$ is given. Similar to the fermionic case, the matrix element ${ }_{N}\left\langle r\left|b_{N}^{\dagger}\right| r^{\prime}\right\rangle_{N}$ appearing in the chain-NRG eq. (47) can be written in terms of the unitary matrices necessary to diagonalize $H_{N}$. The matrix elements ${ }_{N}\left\langle r\left|\sigma_{z}\right| r^{\prime}\right\rangle_{N}$ in eq. (46), however, have to be calculated iteratively. (The technical details are very similar to the fermionic case, see Refs. 1, 2).

With $N_{\mathrm{s}}$ the dimension of $H_{N}$ and $N_{\mathrm{b}}$ the number of basis states in $|s(N+1)\rangle$, we then arrive at a single $\left(N_{\mathrm{s}} \cdot N_{\mathrm{b}}\right) \times\left(N_{\mathrm{s}} \cdot N_{\mathrm{b}}\right)$ matrix for $H_{N+1}\left(r s, r^{\prime} s^{\prime}\right)$. This matrix can be diagonalized using standard routines. From this we obtain the unitary matrices $U_{N+1}(r s, \bar{r})$ and the spectrum of eigenenergies $E_{N+1}(\bar{r})$ so that

$$
H_{N+1}|\bar{r}\rangle_{N+1}=E_{N+1}(\bar{r})|\bar{r}\rangle_{N+1}, \bar{r}=1, \ldots N_{\mathrm{s}} \cdot N_{\mathrm{b}} .
$$

In contrast to the fermionic case, no symmetries can be taken into account to separate the matrix $H_{N+1}\left(r s, r^{\prime} s^{\prime}\right)$ into smaller sub-matrices.

The dimension of $H_{N+1}$ now has to be reduced from $N_{\mathrm{s}} \cdot N_{\mathrm{b}}$ to $N_{\mathrm{s}}$ to allow for a numerical calculation with computation time growing only linearly with $N$. This is achieved with the usual truncation scheme where only the lowest $N_{\mathrm{s}}$ eigenstates of $H_{N+1}$ are kept (for the fermionic case see Refs. 1, 2). These states form the basis $|r\rangle_{N+1}$ for the next step and the iteration continues.

The calculation of correlation functions, such as the spin-spin correlation function $C(\omega)$ in Sec. V, requires the calculation of additional matrix elements ${ }_{N}\left\langle r|\hat{A}| r^{\prime}\right\rangle_{N}$. For more details see Sec. V.

\section{FLOW AND FIXED POINTS}

The iterative numerical diagonalization of the spinboson model as described in the previous section gives

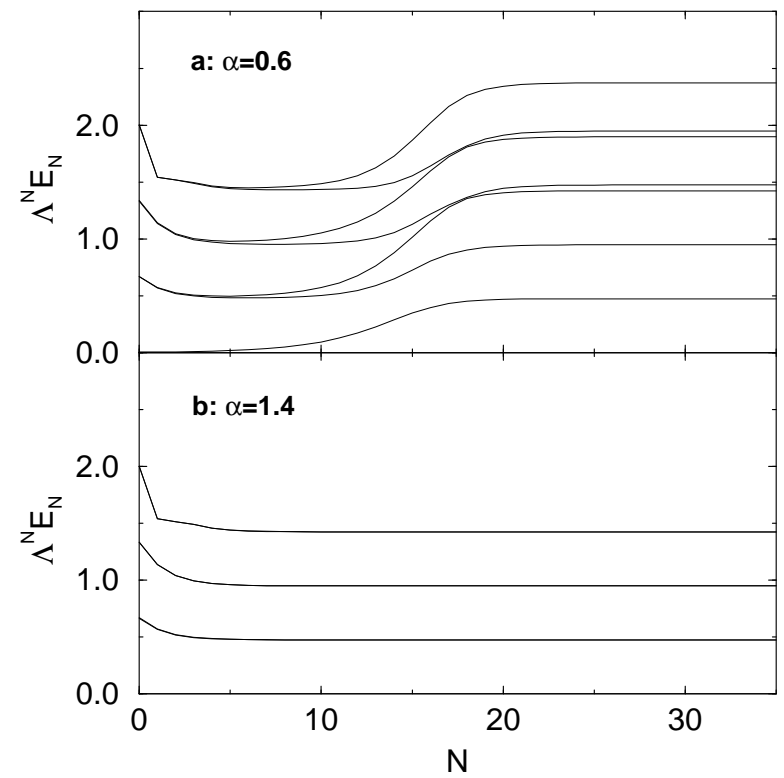

FIG. 5. Flow diagrams calculated with the chain-NRG for the parameters $s=1$ (Ohmic case), $\omega_{c}=1, \epsilon=0, \Delta=0.01$, and $\alpha=0.6$ in (a) and $\alpha=1.4$ in (b). The NRG parameters are $N_{\mathrm{s}}=100, N_{\mathrm{b}}=8$, and $\Lambda=2.0$.

a sequence of many-particle levels $E_{N}(r)\left(r=1, \ldots N_{\mathrm{s}}\right)$. Due to the logarithmic discretization, these energies fall off as $E_{N}(r) \propto \Lambda^{-N}$. NRG flow diagrams can then be constructed by plotting $\Lambda^{N} E_{N}(r)$ versus iteration number $N$.

In this section we focus on those issues which can be directly inferred from the NRG flow diagrams: the appearance of fixed points, the crossover between different fixed points at finite energy or temperature, and quantum phase transitions between the fixed points. Subsections III A-III C deal with the Ohmic spin-boson model; here we also address the issue of convergence. In subsection III D we investigate those features connected to the flow of energy levels which are specific for the sub-Ohmic case.

All results are calculated for cutoff energy $\omega_{c}=1$ and bias $\epsilon=0$; we employ NRG parameter values of $\Lambda=$ $1.8-3.2, N_{\mathrm{b} 0}=100, N_{\mathrm{b}}=4-14, N_{\mathrm{s}}=30-120$.

\section{A. Fixed Points}

Let us first concentrate on results from the chain-NRG for the Ohmic case, $s=1$, and various values of $\Delta$ and $\alpha$.

Fig. 5 shows two NRG flow diagrams for $\Delta=0.01$ and two values for the coupling: $\alpha=0.6$ in Fig. 5a and $\alpha=1.4$ in Fig. 5b. In these diagrams, the rescaled many-particle energies $\Lambda^{N} E_{N}(r)$ are plotted versus the iteration number $N$ with the ground state energy subtracted. Another difference to the fermionic case (apart from the different prefactor $\Lambda^{N}$ instead of $\Lambda^{N / 2}$ ) is the absence of an even-odd effect: in the fermionic case, the 
many-particle spectrum usually oscillates between two sets of energy levels (so that it is more appropriate to speak of a limit cycle than of a fixed point). Plotting the many-particle spectrum either for even or for odd iteration numbers only then gives the flow diagrams as shown in, for example, Refs. 1, 2.

In our bosonic NRG calculations, we can follow the flow typically up to $N=60$ (corresponding to $T \approx 10^{-20}$ for $\Lambda=2.0$ ), then we observe an unphysical runaway which is due to the accumulation of numerical errors in the course of the iteration. As the runaway scale depends on the numerical precision used in the program code, it can be shifted to lower temperatures if needed.

The flow diagrams of Fig. 5 show the existence of two different fixed points: the delocalized fixed point for small $\alpha$ (see Fig. 5a, $N>20$ ) and the localized fixed point for large $\alpha$ (see Fig. 5b, $N>6$ ). These two fixed points are stable and the quantum phase transition between them is discussed further below.

If the value of $\Delta$ is small enough (as in Fig. 5a) the system is close to the localized fixed point in an intermediate range $(4<N<8$ in Fig. 5 a) even for $\alpha$ values below the critical coupling $\alpha_{\mathrm{c}}$. This has direct consequences for thermodynamic properties in the corresponding temperature range (see, for example, Fig. 14). However, the vicinity to the localized fixed point does not imply localization in the sense that a system initially prepared with the impurity spin in one specified direction remains in this spin state under time evolution. For any finite temperature, thermal excitations destroy localization (see Ref. 14).

In Fig. 5a, we also observe a crossover from the localized to the delocalized fixed point which takes place at $N \approx 10-20$. The corresponding crossover scale, $T^{*}$ (which - in the Ohmic case - is equivalent to the renormalized tunnel splitting $\Delta_{\mathrm{r}}$ up to a prefactor), will be discussed in subsection III C.

The flow diagram of Fig. 5a is similar to the one obtained in Ref. 26 (Fig. 1 in Ref. 26), where the mapping of the spin-boson model to the anisotropic Kondo model was employed. The structure of the many-particle levels, however, cannot be directly compared as they reflect the type of bath used in the NRG approach (bosonic in our case, fermionic in Ref. 26).

The spectrum of the delocalized fixed point (Fig. 5a for $N>20$ ) is identical to the spectrum of a spin-boson model with zero coupling between spin and bosons $(\alpha=$ $0)$. The $H_{N}$ for the chain-NRG then take the form:

$$
\begin{aligned}
H_{N, \mathrm{c}} & =\Lambda^{N}\left[H_{\mathrm{loc}}+\sum_{n=0}^{N} \epsilon_{n} b_{n}^{\dagger} b_{n}\right. \\
& \left.+\sum_{n=0}^{N-1} t_{n}\left(b_{n}^{\dagger} b_{n+1}+b_{n+1}^{\dagger} b_{n}\right)\right]
\end{aligned}
$$

In this Hamiltonian, impurity and bath degrees of freedom are completely decoupled and can be diagonalized separately. The spectrum of the impurity part $\left(H_{\mathrm{loc}}=\right.$ $\left.-\Delta \sigma_{x} / 2\right)$ is non-degenerate. The bath part is that of a

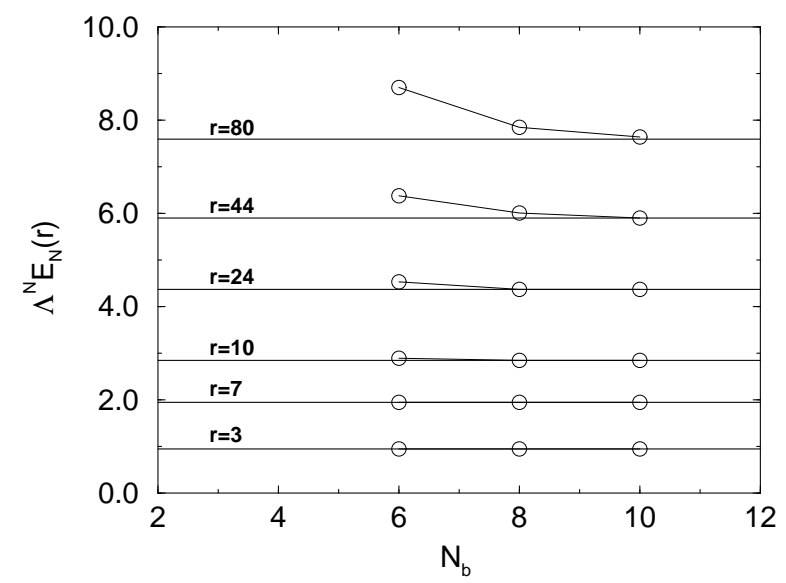

FIG. 6. Comparison between the fixed-point spectra for the delocalized fixed point calculated with the chain-NRG (circles) for various values of $N_{\mathrm{b}}$ and the fixed point spectra constructed from the single particle levels $\bar{\omega}_{n}$ in eq. (50) (solid lines) for a selection of states $E_{N}(r)$. The parameters are $s=1, \Delta=1.0$, and $\alpha<\alpha_{\mathrm{c}}$. The NRG parameters are $N_{\mathrm{s}}=100$, and $\Lambda=2.0$.

free chain of bosons with $N+1$ sites which can be diagonalized exactly:

$$
\sum_{n=0}^{N} \epsilon_{n} b_{n}^{\dagger} b_{n}+\sum_{n=0}^{N-1} t_{n}\left(b_{n}^{\dagger} b_{n+1}+b_{n+1}^{\dagger} b_{n}\right)=\sum_{n=0}^{N} \bar{\omega}_{n} \bar{b}_{n}^{\dagger} \bar{b}_{n} .
$$

Figure 6 shows a comparison between the fixed point spectra for the delocalized fixed point calculated with the chain-NRG (circles) and the fixed-point spectra constructed from the single particle levels $\bar{\omega}_{n}$ in eq. (50) (solid lines). The NRG data are calculated for different $N_{\mathrm{b}}$. The agreement is very good for the first few excitations already for $N_{\mathrm{b}} \approx 6$, while a larger value of $N_{\mathrm{b}}$ is required to correctly reproduce the excitations at higher energies.

While the delocalized fixed point is reached for $\alpha$ smaller than a critical $\alpha_{\mathrm{c}}(\Delta)$, the system is in the localized phase for all $\alpha>\alpha_{\mathrm{c}}(\Delta)$. The localized phase is characterized by a (renormalized) tunneling amplitude $\Delta_{\mathrm{r}}=0$ and a two-fold degenerate ground state. In the language of the (perturbative) renormalization group ${ }^{14,35}$ the localized phase corresponds to a line of fixed points, parametrized by $\alpha$. Interestingly, the fixed-point value of $\alpha$ does not influence the eigenenergies of the many-body fixed-point Hamiltonian, but only its eigenstates, see the discussion in Sec. II. Thus the NRG level spectrum in the entire localized phase is identical to the one for the delocalized fixed point, apart from an additional two-fold degeneracy of all many-particle levels. This feature can be clearly seen in Fig. 5. [Of course, the approach to the localized fixed point depends on the particular value of $\alpha$, consequently the NRG flow on intermediate scales will be different for different $\alpha>\alpha_{\mathrm{c}}(\Delta)$.] 


\section{B. Critical Coupling and Convergence}

The results shown in Fig. 5 indicate the well-known transition between the localized and delocalized fixed points at a critical $\alpha_{\mathrm{c}}(\Delta)^{14,15}$. Due to the KosterlitzThouless nature of this transition, the fixed point at $\alpha=\alpha_{\mathrm{c}}(\Delta)$ is not a new fixed point, but belongs to the localized phase instead.

On approaching the transition from the delocalized side, we find, as expected, that the crossover scale vanishes $\operatorname{as}^{38} \ln T^{*} \propto 1 /\left(\alpha_{\mathrm{c}}-\alpha\right)$, see Fig. 11 below. We use this dependence to determine the value of $\alpha_{\mathrm{c}}$ from numerically calculated data for $T^{*}(\alpha)$ via a non-linear fit. [Note that on the localized side of the transition a low-energy scale only shows up in the flow towards the fixed point, i.e., in corrections to the fixed-point values of observables; thus the critical $\alpha_{\mathrm{c}}(\Delta)$ is easier obtained via extrapolation from the delocalized side.]

As already discussed in Ref. 13 (see Fig. 2 in Ref. 13), the value of $\alpha_{\mathrm{c}}$ also depends on the NRG parameters $\Lambda$, $N_{\mathrm{b}}$, and $N_{\mathrm{s}}$. Figures 7a-c show the characteristic dependence for the Ohmic case, $s=1$, and two values of $\Delta$ in Fig. 7c. Keeping $\Lambda$ fixed, we observe a rapid convergence of $\alpha_{\mathrm{c}}$ with increasing $N_{\mathrm{s}}$ (Fig. 7a) and $N_{\mathrm{b}}$ (Fig. 7b). Note that we did not observe a transition to the delocalized phase for $N_{\mathrm{b}} \leq 4$, even for very large values of $\alpha$. As expected from the iterative diagonalization scheme, the values of $N_{\mathrm{s}}$ and $N_{\mathrm{b}}$ necessary for convergence increase with decreasing $\Lambda$ (see Fig. 7c). The converged data for $\alpha_{\mathrm{c}}(\Lambda)$ show a linear $\Lambda$ dependence in the range $1.8<\Lambda<3$, with a deviation of about $15 \%$ at $\Lambda=2$ from the extrapolated $\Lambda \rightarrow 1$ value.

We find that the slope in $\alpha_{\mathrm{c}}(\Lambda)$ is independent of $\Delta$ which is connected to fact that the logarithmic discretization systematically underestimates the spectral weight contained in $J(\omega)$ (for a discussion of this point in the fermionic case, see eq. (5.42) in Ref. 2; for the soft-gap Anderson model see Fig. 4 in Ref. 36).

The extrapolated values $\alpha_{\mathrm{c}}(\Delta, \Lambda \rightarrow 1)$ for the Ohmic case are summarized in Fig. 8. In the limit of small $\Delta$, the NRG result is in good agreement with the well established value $\alpha_{\mathrm{c}}(s=1, \Delta \rightarrow 0)=1$. We estimate the error in $\alpha_{\mathrm{c}}$ to be of the order of 0.02 which is due to the various extrapolations just described. The solid line in Fig. 8 shows a linear fit to the numerical data which gives $\alpha_{\mathrm{c}}(\Delta)=0.99+0.53 \Delta$. This is consistent with the RG result $\alpha_{\mathrm{c}}=1+\mathcal{O}\left(\Delta / \omega_{c}\right)^{14}$.

\section{Scaling}

We expect to observe scaling behavior in all physical properties for fixed $\Delta$ and $\alpha \rightarrow \alpha_{\mathrm{c}}(\Delta)$ and for fixed $\alpha$ and $\Delta \rightarrow 0$. Such a scaling can already be identified on the level of the flow of the many-particle energies. An example is shown in Fig. 9 for fixed $\alpha=0.6$ and various values of $\Delta$. In this way we can easily determine the
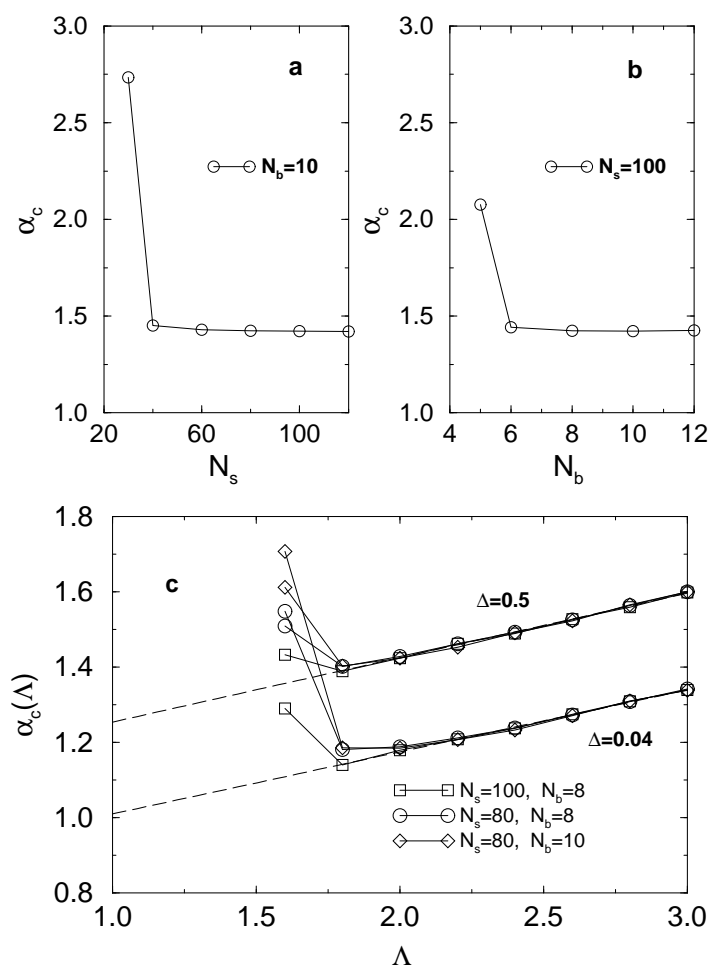

FIG. 7. Dependence of the critical coupling $\alpha_{\mathrm{c}}$ on the NRG parameters $N_{\mathrm{s}}, N_{\mathrm{b}}$, and $\Lambda$ for the Ohmic case; (a) dependence on $N_{\mathrm{s}}$ for fixed $N_{\mathrm{b}}=10$; (b) dependence on $N_{\mathrm{b}}$ for fixed $N_{\mathrm{s}}=100(\Lambda=2.0$ for both (a) and (b)); (c) $\Lambda$-dependence of $\alpha_{\mathrm{c}}$ for two values of $\Delta$, and various NRG parameters $N_{\mathrm{b}}$ and $N_{\mathrm{s}}$. The dashed lines are linear fits to the $N_{\mathrm{b}}=8$ and $N_{\mathrm{s}}=100$ data in the range $1.8 \leq \Lambda \leq 3$.

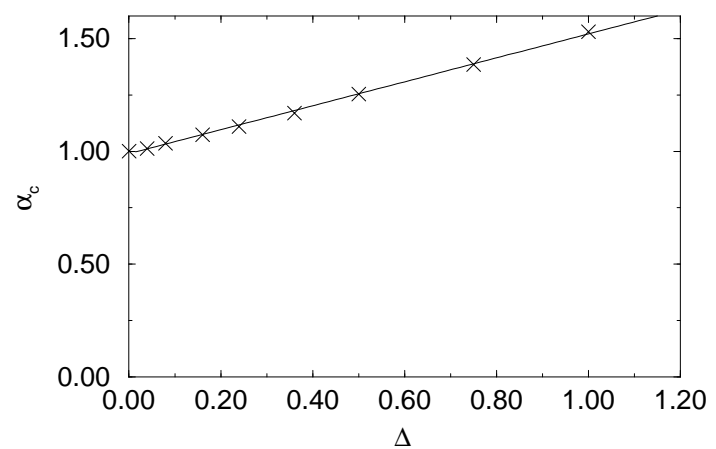

FIG. 8. Dependence of the extrapolated value $\alpha_{\mathrm{c}}(\Lambda \rightarrow 1)$ on the parameter $\Delta$ for the Ohmic case $s=1$. The crosses are the numerical data and the solid line is a linear fit which gives $\alpha_{\mathrm{c}}(\Delta)=0.99+0.53 \Delta$. The NRG parameters are $N_{\mathrm{s}}=100$ and $N_{\mathrm{b}}=8$.

crossover scale $T^{*}$ for the crossover from the localized to the delocalized fixed point (there is only a single lowenergy scale):

$$
T^{*}=\text { const. } \times \Lambda^{-N^{*}},
$$

where we define $N^{*}$ as the value of $N$ where the first excited state reaches the value $E_{N}=0.3$. Note that a change of this (arbitrary) value can be absorbed in a 


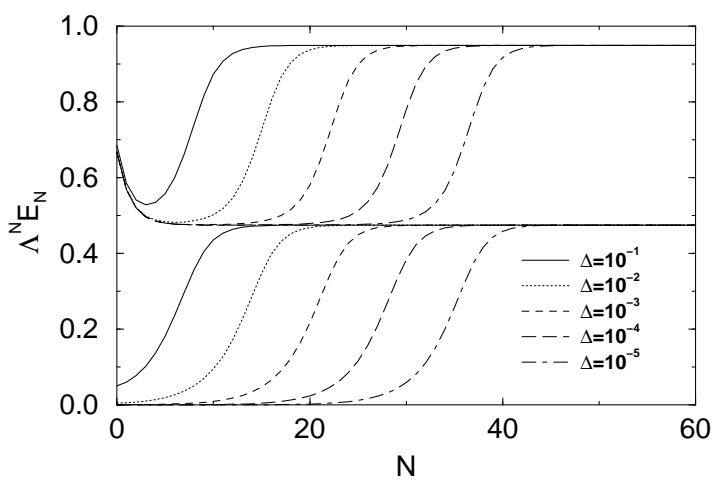

FIG. 9. Scaling of the flow of the many-particle levels $E_{N}(r)$ for fixed $\alpha=0.6, s=1$, and various values of $\Delta$. The NRG parameters are $N_{\mathrm{s}}=100, N_{\mathrm{b}}=8$, and $\Lambda=2.0$.

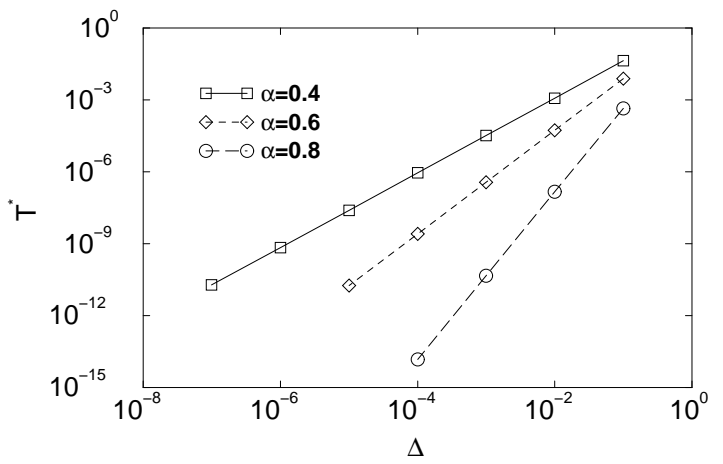

FIG. 10. Dependence of the crossover temperature $T^{*}$ on $\Delta$ for $s=1$ and fixed values of $\alpha$. The exponents in $T^{*} \propto \Delta^{x}$ are $x \approx 1.55$ for $\alpha=0.4, x \approx 2.15$ for $\alpha=0.6$, and $x \approx 3.49$ for $\alpha=0.8$. The NRG parameters are $N_{\mathrm{s}}=100, N_{\mathrm{b}}=8$, and $\Lambda=2.0$.

change of the prefactor in eq. (51); this reflects the fact that a temperature scale can only be defined up to a constant prefactor anyway.

In the scaling regime, the dependence of $T^{*}$ on $\alpha$ and $\Delta$ is given by ${ }^{14,38}$

$$
T^{*} \propto \Delta^{1 /\left(\alpha_{c}-\alpha\right)} .
$$

As shown in Figs. 10 and 11, the NRG results are in agreement with eq. (52).

\section{Flow for Sub-Ohmic Baths}

As already mentioned in Sec. II F, the chain-NRG with a basis of undisplaced oscillators as in eq. (28) is sufficient for the Ohmic and super-Ohmic case. Let us now turn to the sub-Ohmic case where we expect problems with the chain-NRG when the system is flowing to the localized fixed point. Figure 12 shows a typical flow diagram of the many-particle energies, calculated with the star-NRG for $s=0.8$ and a couple of $\alpha$ values close to the quantum critical point $\alpha_{\mathrm{c}}=0.40294$.

In contrast to the Ohmic case, the transition in the sub-Ohmic case is characterized by a new fixed point, the

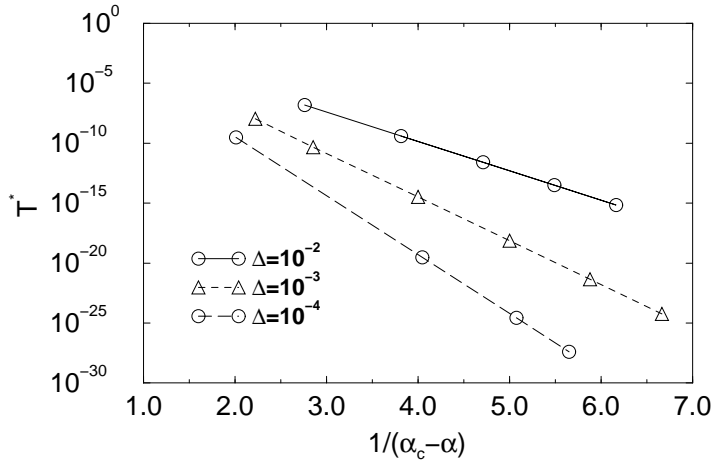

FIG. 11. Dependence of the crossover temperature $T^{*}$ on $\alpha$ for $s=1$ and fixed values of $\Delta$ (data for $\Delta=10^{-3}$ and $\Delta=10^{-4}$ same as in Fig. 4b of Ref. 13). The values for the critical coupling are $\alpha_{\mathrm{c}}=1.162$ for $\Delta=10^{-2}, \alpha_{\mathrm{c}}=1.150$ for $\Delta=10^{-3}$, and $\alpha_{\mathrm{c}}=1.147$ for $\Delta=10^{-4}$. The NRG parameters are $N_{\mathrm{s}}=100, N_{\mathrm{b}}=8$, and $\Lambda=2.0$.

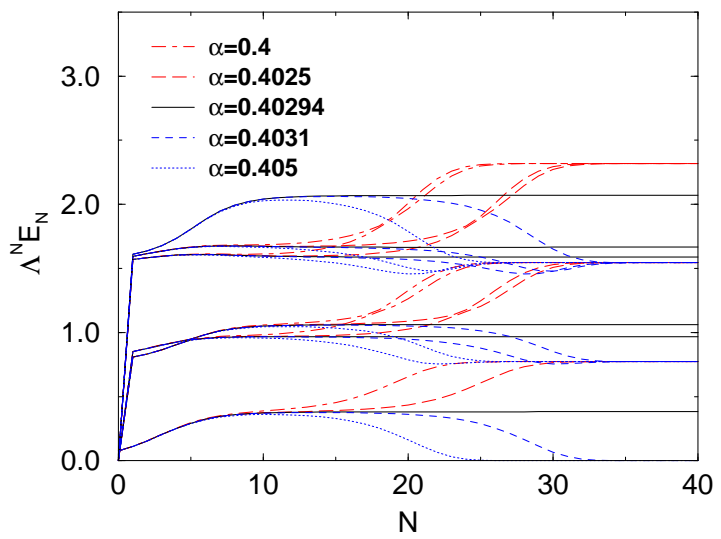

FIG. 12. (Color online) Flow diagram of the lowest lying many-particle energies calculated with the star-NRG for the sub-Ohmic case $(s=0.8, \Delta=0.1)$, using displaced oscillators as optimized basis. The critical value is $\alpha_{\mathrm{c}}=0.40294$. The NRG parameters are $N_{\mathrm{s}}=80, N_{\mathrm{b}}=8$, and $\Lambda=2.0$.

quantum critical fixed point, with a level structure which is different from both the localized and the delocalized fixed points. For any $\alpha \neq \alpha_{\mathrm{c}}$ there is a finite crossover scale $T^{*}$ for the crossover to the localized fixed point (for $\alpha>\alpha_{\mathrm{c}}$ ) and to the delocalized fixed point (for $\alpha<\alpha_{\mathrm{c}}$ ). The crossover scale can be defined in a similar way as in Sec. III C. A further analysis of the dependence of $T^{*}$ on $\left|\alpha-\alpha_{\mathrm{c}}\right|$ gives the critical exponents. Their $s$-dependence has been shown already in Fig. 5a of Ref. 13. A detailed investigation of the critical properties of the sub-Ohmic spin-boson model will appear elsewhere.

Here we focus on the level structure of the localized and delocalized fixed point in Fig. 12. Both fixed points have exactly the same level structure apart from an additional two-fold degeneracy of all levels of the localized fixed point. This is evident from Fig. 12 (levels for $\alpha>\alpha_{\mathrm{c}}$ and $\alpha<\alpha_{\mathrm{c}}$ converge to the same spectrum) and also follows from the discussion of Sec. II E. However, the proper description of the localized fixed point can only 


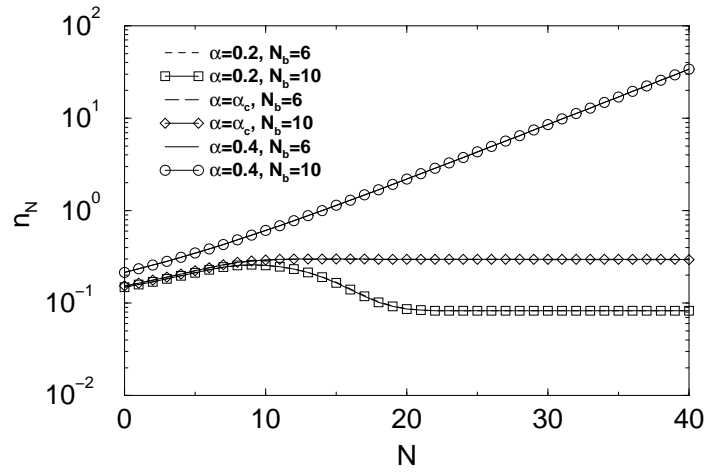

FIG. 13. Dependence of the expectation value $n_{N}=\left\langle b_{N}^{\dagger} b_{N}\right\rangle$ on the iteration number $N$ for $s=0.8$, $\Delta=0.01, \alpha=0.2<\alpha_{\mathrm{c}}$ (squares), $\alpha=\alpha_{\mathrm{c}}=0.21488785$ (diamonds), and $\alpha=0.4>\alpha_{\mathrm{c}}$ (circles), and two different values of $N_{\mathrm{b}}$, calculated with the star-NRG.

be achieved using an optimized basis with displacements calculated as discussed in Sec. II F and Appendix B. Using a basis of undisplaced oscillators $(\theta=0)$ leads to an incorrect level structure. This can be seen in the upper right-hand panel of Fig. 3 in Ref. $13\left(s=0.6, \alpha>\alpha_{\mathrm{c}}\right)$ where the basis (28) was used. The resulting fixed point levels are therefore not the same as the one for $\alpha<\alpha_{\mathrm{c}}$ in the upper left-hand panel of Fig. 3 in Ref. 13.

As mentioned in Sec. IIF, we did not yet succeed to implement the concept of displaced oscillators in the chain-NRG, so the proper description of the localized fixed point for $s<1$ is presently only possible with the star-NRG. Fortunately, the problems of the chain-NRG only show up when the flow is approaching the localized fixed point. We can therefore safely extract all the critical properties such as critical exponents from the chainNRG, as has been done in Ref. 13.

On the other hand, the use of a basis of displaced oscillators within the star-NRG solves the problem of the boson-number divergence (see Sec. IIF). This is illustrated in Fig. 13 where the dependence of the expectation value $n_{N}=\left\langle b_{N}^{\dagger} b_{N}\right\rangle$ is shown for three values of $\alpha$ $\left(\alpha=0.2<\alpha_{\mathrm{c}}, \alpha=\alpha_{\mathrm{c}}=0.21488785\right.$, and $\left.\alpha=0.4>\alpha_{\mathrm{c}}\right)$ and two values of $N_{\mathrm{b}}$. For all values of $\alpha$ we observe a rapid convergence with $N_{\mathrm{b}}$, similar to the convergence shown for $\alpha<\alpha_{\mathrm{c}}$ and $\alpha=\alpha_{\mathrm{c}}$ in Fig. 3. The difference here is that the data converge with $N_{\mathrm{b}}$ also for $\alpha>\alpha_{\mathrm{c}}$ which can not be achieved by using the basis (25), see Fig. 3. Furthermore, the expectation value $n_{N}$ diverges exponentially with $N$ for $\alpha>\alpha_{\mathrm{c}}$, as expected from the discussion in Sec. IIE. A diverging boson number itself is therefore not a problem for the bosonic NRG, provided a proper optimized basis is chosen.

Finally, a few words on the limitations of the starNRG. Whereas the localized fixed point is described correctly, the star-NRG seems to fail in other respects: the low-energy flow to the delocalized fixed point appears incorrect, and critical exponents of the quantum phase transition deviate from the chain-NRG results (and from analytically known values). We do not yet fully understand this problem, but it might be connected to truncation errors which affect the star-NRG in a completely different way as the chain-NRG. (The idea is that the truncation somehow affects the character of the impurity operator to which the added bosonic site couples in each step.) The precise characterization of this problem and its possible solution are left for future studies.

\section{THERMODYNAMIC QUANTITIES}

In this section, we describe how thermodynamic quantities can be extracted from the flow of many-particle levels $E_{N}(r)$ which are calculated with the bosonic NRG. Starting from the $E_{N}(r)$ there is no difference (from a technical point of view) between the fermionic and the bosonic case (for the fermionic case see, for example, Refs. 2, 37). Nevertheless, for completeness we include a brief discussion of the technical details here. We show results for the impurity contribution to the entropy and the specific heat in the Ohmic case (using the chain-NRG with basis (28)). The Ohmic case has been studied in detail in Refs. 26, 39 (for earlier work on thermodynamic properties see Refs. 14, 40, 41). The agreement with the results from Refs. 26, 39 is excellent which again confirms the reliability of the bosonic NRG for the investigation of quantum impurity models involving a bosonic bath. A few comments on thermodynamic properties in the subOhmic case are given at the end of this section.

Consider the spectrum of many-particle energies $E_{i}$ of a discretized version of the spin-boson model (not necessarily the discretized Hamiltonians (11) and (14) used in the bosonic NRG). The grand canonical partition function, $Z=\operatorname{Tr} e^{-\beta(H-\mu N)}$, reduces to

$$
Z=\sum_{i} e^{-\beta E_{i}}
$$

as the chemical potential $\mu$ is set to zero (we are interested in gapless spectral functions $J(\omega)$ ). Free energy $F$ and entropy $S$ are then given by

$$
F=-T \ln Z \text { and } S=-\frac{\partial F}{\partial T} .
$$

(We set $k_{\mathrm{B}}=1$.) The impurity contribution to the entropy is

$$
S_{\mathrm{imp}}=S-S_{0}
$$

where $S$ is the entropy of the full system and $S_{0}$ the entropy of the system without impurity.

Before we discuss the full temperature dependence of $S_{\mathrm{imp}}(T)$, let us focus on the value of $S_{\mathrm{imp}}$ at the localized and delocalized fixed points: $S_{\mathrm{imp}, \mathrm{L}}$ and $S_{\mathrm{imp}, \mathrm{D}}$. It is well known that $S_{\mathrm{imp}, \mathrm{L}}=\ln 2$ and $S_{\mathrm{imp}, \mathrm{D}}=0^{26,39}$, but it might not be obvious that these values can be directly extracted from the many-particle spectra at the fixed points. 
In Sec. III A we already showed that the fixed point spectrum of the delocalized fixed point is the same as the one of a free bosonic chain, which is nothing else but the system without impurity. This implies that for the delocalized fixed point

$$
E_{i}=E_{i, 0}+\Delta E
$$

with $E_{i}\left(E_{i, 0}\right)$ the many-particle energies of the system with (without) impurity and $\Delta E$ a constant shift independent of $i$. It is clear that this equation cannot hold for all levels, it is only valid for energies sufficiently below the crossover scale to the fixed point.

Equation (56) directly leads to the proof of $S_{\mathrm{imp}, \mathrm{D}}=0$ : we have $Z_{\mathrm{D}}=\exp [-\beta \Delta E] Z_{0}$, and from this $F_{\mathrm{D}}=F_{0}+$ $\Delta E$. The energy shift drops out in the derivative so that $S_{\mathrm{D}}=S_{0}$ and the impurity contribution to the entropy at the delocalized fixed point is given by $S_{\text {imp,D }}=0$.

In a similar way one can easily prove that $S_{\mathrm{imp}, \mathrm{L}}=\ln 2$ : in this case we have

$$
Z_{\mathrm{L}}=2 \sum_{i} e^{-\beta E_{i}}, \quad E_{i}=E_{i, 0}+\Delta E,
$$

with the factor of 2 due to the additional double degeneracy of all many-particle levels at the localized fixed point. This gives $Z_{\mathrm{L}}=2 \exp [-\beta \Delta E] Z_{0}$, and from this $F_{\mathrm{L}}=-T \ln 2+F_{0}+\Delta E$ and $S_{\mathrm{L}}=\ln 2+S_{0}$, corresponding to $S_{\mathrm{imp}, \mathrm{L}}=\ln 2$. From this discussion it follows that $S_{\mathrm{imp}, \mathrm{L}}=\ln 2$ and $S_{\mathrm{imp}, \mathrm{D}}=0$ independent of the exponent $s$ in the spectral function $J(\omega)$.

For any finite $\Delta$ and $\alpha$, the values $S_{\text {imp,L }}=\ln 2$ and $S_{\mathrm{imp}, \mathrm{D}}=0$ are strictly valid only in the limit $T \rightarrow 0$. Note that a proper definition of these zero-point entropies requires the correct order of limits: the thermodynamic limit has to be taken before the limit $T \rightarrow 0$. With the order of the limits reversed, the zero-point entropy would be equal to $\ln d_{\mathrm{g}}$, with $d_{\mathrm{g}}$ the degeneracy of the ground state. Although this happens to give the same values for $S_{\mathrm{imp}, \mathrm{L}}$ and $S_{\mathrm{imp}, \mathrm{D}}$ in the case studied here, this equivalence is not generally valid. (This can be seen, for example, in the NRG calculations for the single-impurity Anderson model ${ }^{2}$ where the degeneracy of the ground state oscillates between 1 for even and 4 for odd iterations when the system approaches the fixed point of a screened spin, which has $S_{\mathrm{imp}}=0$. Also, any non-trivial quantum critical fixed point is expected to have a residual entropy which is not $\ln d_{\mathrm{g}}$ with integer $d_{\mathrm{g}}$.)

The impurity contribution to the entropy is close to a fixed point value also when the system is close to this fixed point in an intermediate range of the flow diagram. From Fig. 5a we can therefore immediately see that the temperature dependence of $S_{\mathrm{imp}}(T)$ contains a crossover from a high-temperature value $S_{\mathrm{imp}} \approx S_{\mathrm{imp}, \mathrm{L}}=\ln 2$ to the low-temperature value $S_{\mathrm{imp}}(T \rightarrow 0)=S_{\mathrm{imp}, \mathrm{D}}=0$; provided the flow is to the delocalized fixed point. The detailed behavior of $S_{\mathrm{imp}}(T)$ in the crossover region requires a numerical calculation as described below.
In the bosonic NRG, we do not have access to the full spectrum of many-particle energies $E_{i}$ as used in eq. (53). Instead, the iterative procedure results in a sequence of many-particle energies $E_{N}(r)$ with iteration number $N$ and $r=1, \ldots N_{\mathrm{s}}$. According to the discussion in Refs. 1, 2 , each of the sets of many-particle energies is assumed to be a good description of the system for a certain temperature $T_{N}$ with

$$
T_{N}=x \omega_{c} \Lambda^{-N}
$$

with $x$ a dimensionless constant of the order of 1 , chosen such that $T_{N}$ lies within the spectrum $E_{N}(r)$.

For each iteration step $N$, the partition function is calculated for the temperature $T_{N}$ :

$$
Z_{N}=\sum_{r} e^{-E_{N}(r) / T_{N}}
$$

In addition, the internal energy at iteration step $N$ for the temperature $T_{N}$ is calculated as

$$
E_{N}=\frac{1}{Z_{N}} \sum_{r} E_{N}(r) e^{-E_{N}(r) / T_{N}}
$$

This is the information we have available for the numerical calculation of thermodynamic properties.

One possibility to proceed is to calculate the free energy $F_{N}=-T_{N} \ln Z_{N}$ for each iteration step, and from this the entropy $S=-\frac{\partial F}{\partial T}$ via a discrete differentiation. This procedure has been shown to give good results in the fermionic case (see, for example, Ref. 42). It requires, however, a precise calculation of the difference of the ground state energies between subsequent steps; this appears to introduce some errors in the calculations within the bosonic NRG. (In general, the bosonic NRG is less accurate in the calculation of thermodynamic properties as compared to the fermionic NRG because we cannot keep as many states as in the fermionic case.)

Therefore, we use an alternative approach in which the entropy $S_{N}$ at iteration step $N$ for the temperature $T_{N}$ is calculated via

$$
S_{N}=\frac{E_{N}}{T_{N}}+\ln Z_{N} .
$$

This approach avoids the discrete differentiation, and does not require the knowledge of the ground state energies.

Let us now discuss the results for entropy and specific heat calculated with the bosonic NRG using the method just described. Figure 14 shows the temperature dependence of the impurity contribution to the entropy, $S_{\mathrm{imp}}(T)$, for $\alpha=1 / 3, s=1$ (Ohmic case), and various values of $\Delta$. We observe a crossover from the hightemperature value $S_{\mathrm{imp}}=\ln 2$ to the low-temperature value $S_{\text {imp }}=0$ at a crossover scale $T^{*}$, which is the same as the one introduced in Sec. III C. The crossover scale decreases with decreasing $\Delta$ in agreement with eq. (52). 


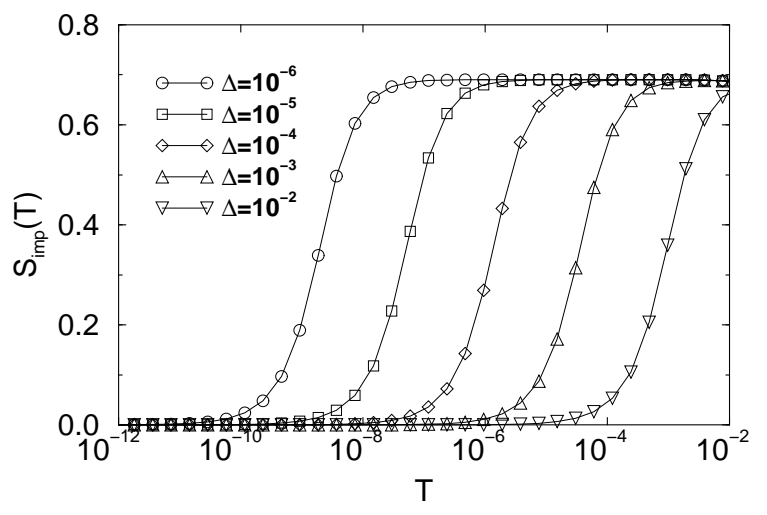

FIG. 14. Temperature dependence of the impurity contribution to the entropy, $S_{\mathrm{imp}}(T)$, for $\alpha=1 / 3, s=1$ (Ohmic case), and various values of $\Delta$.

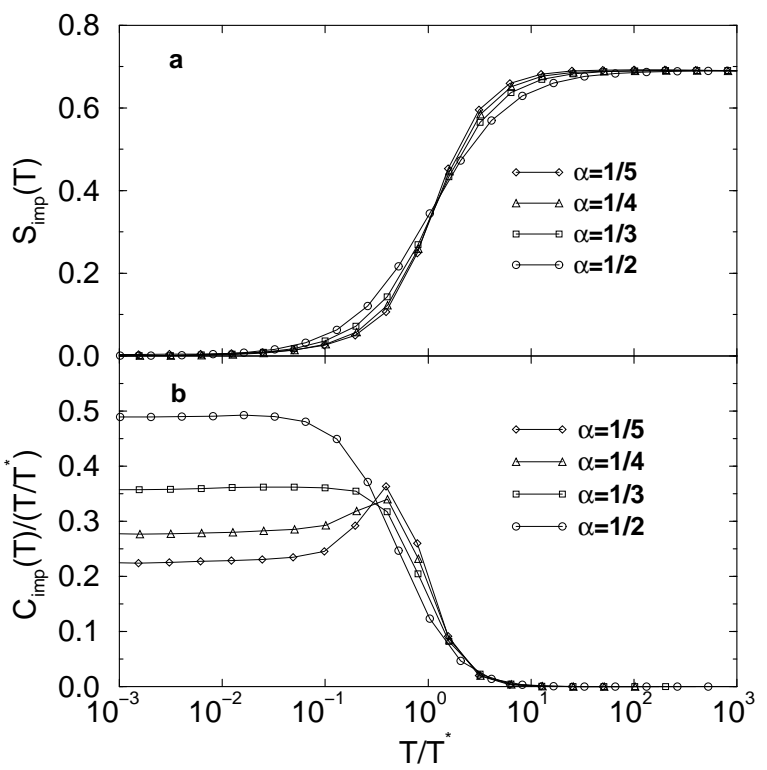

FIG. 15. (a) Scaling curves of the impurity contribution to the entropy, $S_{\mathrm{imp}}(T)$, for $s=1$ (Ohmic case), and various values of $\alpha$; (b) Scaling curves of the impurity contribution to the specific heat, $C_{\mathrm{imp}}(T) /\left(T / T^{*}\right)$, for the same parameters as in (a).

Note the similarity of Fig. 14 to Fig. 9 for the scaling of the energy levels, a similarity which is simply due to the relation between $S_{\mathrm{imp}}(T)$ and the flow of the manyparticle levels.

As briefly mentioned in Sec. III A, the vicinity to the localized fixed point for early iterations (which results in the high-temperature value $\left.S_{\mathrm{imp}}(T) \approx \ln 2\right)$ does not imply localization. The value of $S_{\mathrm{imp}}(T)$ for high temperatures is due to the fact that for temperatures $T \gg \Delta$ both states of the two-state system contribute equally to the thermodynamics. Note also the similarity to $S_{\text {imp }}(T)$ in the Kondo model: there the high-temperature phase is that of a local moment with both spin $\uparrow$ and $\downarrow$ configurations contributing to the entropy (a temperature dependence of $S_{\mathrm{imp}}(T)$ as in Fig. 14 might therefore ap-

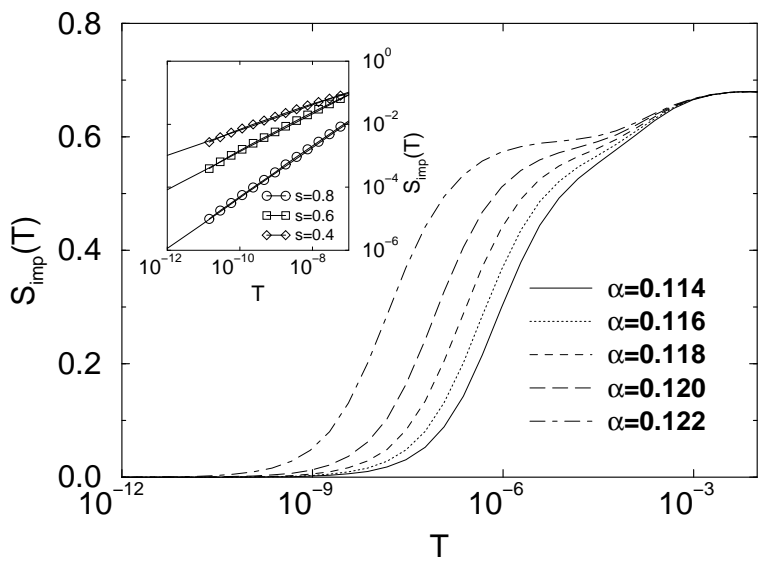

FIG. 16. Temperature dependence of the impurity contribution to the entropy, $S_{\mathrm{imp}}(T)$, in the sub-Ohmic case for various values of $\alpha$ and $s=0.8$ (main panel) and various values of $s$ (inset). The coupling $\alpha$ is below $\alpha_{\mathrm{c}}$ so that the flow is to the delocalized phase for all parameters in this figure. Lines with symbols in the inset are data from the bosonic NRG and solid lines are fits assuming a power-law, $S_{\text {imp }}(T) \propto T^{s}$.

pear more natural in the Kondo model but, of course, it is also valid here).

The scaling behavior of $S_{\mathrm{imp}}(T)$ for fixed $\alpha=1 / 3$ and various $\Delta$ is obvious and is shown in Fig. 15a together with the scaling curves for $\alpha=1 / 5,1 / 4$, and $1 / 2$. The agreement with the exact results from the Bethe Ansatz calculations in Ref. 39 is very good (see Fig. 7a in Ref. 39), in particular for the $\alpha$-dependence of the scaling curves.

The temperature dependence of the specific heat, $C_{\mathrm{imp}}(T)$, is calculated via $C_{\mathrm{imp}}(T) / T=\partial S_{\mathrm{imp}}(T) / \partial T$. Here we cannot avoid the discrete differentiation of $S_{\mathrm{imp}}(T)$. The scaling of $S_{\mathrm{imp}}(T)$ implies a scaling of $C_{\mathrm{imp}}(T) / T$ as shown in Fig. 15b. This figure is also very similar to previous calculations (see Fig. 2 in Ref. 26 from the NRG via mapping to the anisotropic Kondo model and Fig. 7b in Ref. 39 using the Bethe Ansatz), and we find the same characteristic features here: a linear specific heat $C \propto T$ for low temperatures, a peak in $C / T$ at $T \approx T^{*}$ for small dissipation $\alpha<0.3$ in contrast to the monotonous decrease of $C / T$ for large dissipation $\alpha>0.3$, and a characteristic crossing point of all the $C / T$ scaling curves.

Similar to the NRG calculations in Ref. 26, the thermodynamic quantities can only be calculated on a discrete mesh of temperatures given by eq. (58). This strongly limits the resolution of the peak in $C / T$ for $\alpha<0.3$, in contrast to the Bethe Ansatz calculations of Ref. 39.

The physics in the sub-Ohmic case is much richer, due to the appearance of a line of quantum critical points ${ }^{13}$. This is reflected in the behavior of the entropy and the specific heat. For the results of $S_{\mathrm{imp}}(T)$ and $C_{\mathrm{imp}}(T)$ close to the quantum critical points we refer to a subsequent publication. Here we focus on the flow to the delocalized phase. 
Figure 16 shows the temperature dependence of the impurity contribution to the entropy, $S_{\text {imp }}(T)$, in the subOhmic case, $s=0.8$, for various values of $\alpha$ below the critical value $\alpha_{\mathrm{c}} \approx 0.125$. For $\alpha$ close to $\alpha_{\mathrm{c}}$ we observe a two stage quenching of the entropy of the free moment (the quantum critical point has a non-trivial zero-point entropy of $S_{\mathrm{qcp}}(T \rightarrow 0) \approx 0.6$ for $\left.s=0.8\right)$. As expected, the temperature scale for the crossover to the delocalized fixed point increases with the distance from the critical point. The low-temperature behavior of $S_{\mathrm{imp}}(T)$ for $\alpha<\alpha_{\mathrm{c}}$ is given by $S_{\text {imp }}(T) \propto T^{s}$ which can be seen more clearly in the inset of Fig. 16 where $S_{\text {imp }}(T)$ is plotted for various values of $s$. This behavior is in agreement with the calculations of Ref. 40, where $C(T) \propto T^{s}$ was found for the slightly asymmetric $(\epsilon \neq 0)$ sub-Ohmic spin-boson model. [While the finite $\epsilon$ turns the quantum phase transition into a smooth crossover, it does not influence the qualitative low-energy behavior in the delocalized phase.]

The data in Fig. 16 are calculated with the chain-NRG. The results from the star-NRG look similar (they give, in particular the correct value $S_{\mathrm{imp}}(T \rightarrow 0)=\ln 2$ if the flow is to the localized phase). We observe, however, a lowtemperature behavior for $S_{\mathrm{imp}}(T)$ which is different from the correct form, $S_{\mathrm{imp}}(T) \propto T^{s}$. As briefly mentioned in Sec. III D, the reason for this failure of the star-NRG is presently not clear but probably due to truncation errors.

Despite these deficiencies, the bosonic NRG is a reliable tool for the calculation of thermodynamic properties in a wide range of parameters and the comparison with well-established results is very promising. Thermodynamic properties in the quantum critical region will be discussed in a separate publication.

\section{DYNAMIC QUANTITIES}

The calculation of dynamic properties is straightforward within the bosonic NRG and proceeds in a very similar way (from a technical point of view) as in the fermionic case. The typical problems such as the combination of information from different iteration steps and the broadening of the discrete spectra have been discussed already in the literature (see, for example, Refs. 43-46, 12) and need not be repeated here.

\section{A. Dynamical Spin Correlations}

One important dynamic quantity of interest in the spin-boson model is the spin-spin correlation function (spin autocorrelation function)

$$
C(\omega)=\frac{1}{2 \pi} \int_{-\infty}^{+\infty} e^{i \omega t} C(t) \mathrm{d} t
$$

with $C(t)=\frac{1}{2}\left\langle\left[\sigma_{z}(t), \sigma_{z}\right]_{+}\right\rangle$. We only consider equilibrium correlation functions, in general for finite temper- atures, but the focus here is on $T=0$ so that the expectation value $\langle\ldots\rangle$ has to be taken with respect to the ground state.

For a discrete Hamiltonian, the spin-spin correlation function at $T=0$ can be written as

$$
C(\omega)=\frac{1}{2} \sum_{n}\left|\left\langle 0\left|\sigma_{z}\right| n\right\rangle\right|^{2} \delta\left(\omega+\epsilon_{0}-\epsilon_{n}\right) \quad, \omega>0,
$$

with $C(\omega)=C(-\omega)$. Note that with the above definition of $C(t)$, the quantity $C(\omega)$ is purely real and related to the imaginary part of the spin-susceptibility $\chi(\omega)$ via $C(\omega)=\frac{1}{2} \pi|\operatorname{Im} \chi(\omega)|$ (see also eq. (3.96) in Ref. 14).

Due to the truncation in the course of the iterative diagonalization, we cannot calculate $C(\omega)$ simultaneously for all energy scales. Instead, the correlation function is calculated for each cluster of length $N$ (which gives information on energy scales of the order of $\Lambda^{-N}$ ) and this information has to be added up properly. Finally, the discrete spectrum has to be broadened which results in continuous curves for $C(\omega)$ as shown, for example, in Fig. 17.

These technical issues are dealt with using the approach described in Ref. 12; to broaden the spectra, we use a Gaussian on a logarithmic scale (see eq. (8) in Ref. 12) with broadening parameter $b=0.7$.

We also define the correlation function $S(\omega)$ as

$$
S(\omega)=\frac{2 C(\omega)}{\omega^{s}}
$$

The static spin-susceptibility $\chi$ is defined as

$$
\chi=2 \frac{\partial\left\langle\sigma_{z}\right\rangle}{\partial \epsilon} \quad, \quad\left\langle\sigma_{z}\right\rangle=\left\langle 0\left|\sigma_{z}\right| 0\right\rangle .
$$

It is related to $C(\omega)$ via

$$
\chi=4 \int_{0}^{\infty} \frac{C(\omega)}{\omega} \mathrm{d} \omega,
$$

and, using eq. (63), can be written in the form

$$
\chi=2 \sum_{n} \frac{\left|\left\langle 0\left|\sigma_{z}\right| n\right\rangle\right|^{2}}{\epsilon_{n}-\epsilon_{0}} .
$$

Here, we calculate the susceptibility according to eq. (67).

The bosonic NRG allows the calculation of dynamic properties in a wide range of frequencies so that the functional dependence of $C$ on the frequency $\omega$ (such as a power-law $\left.C(\omega) \propto \omega^{s}\right)$ can be easily extracted. However, the exponent of the calculated $C(\omega)$ has a deviation from the expected value $s$ (for the flow to the delocalized fixed point) of about $2 \%$. To extract the correct prefactor of $C(\omega)$ (which we use to compare with the exact results at the Toulouse point and to check the Shiba relation), we need to redefine the quantity $S(\omega)$ as

$$
S(\omega)=\frac{2 C(\omega)}{\omega^{\delta}}
$$




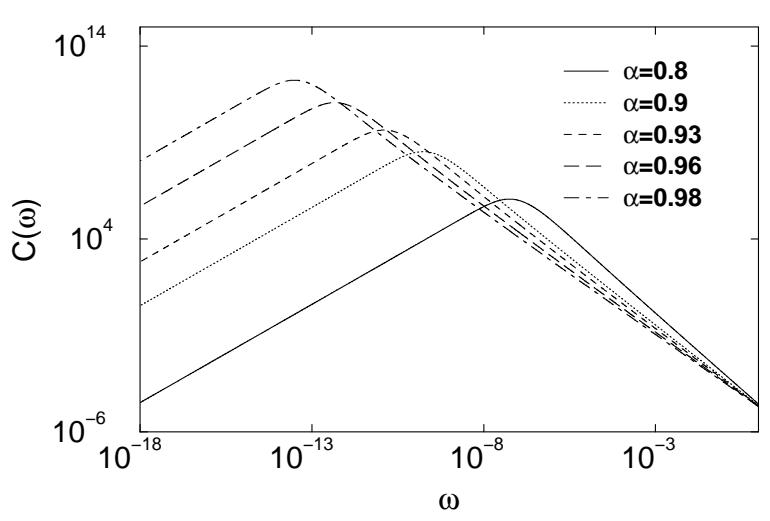

FIG. 17. Spin-spin correlation function $C(\omega)$ calculated for the Ohmic case, $s=1, \Delta=0.01$, and various values of $\alpha<\alpha_{\text {c }}$ close to the transition. For small frequencies, $C(\omega) \propto \omega$, whereas for higher frequencies we observe a divergence, $C(\omega) \propto \omega^{-1}$, with logarithmic corrections.

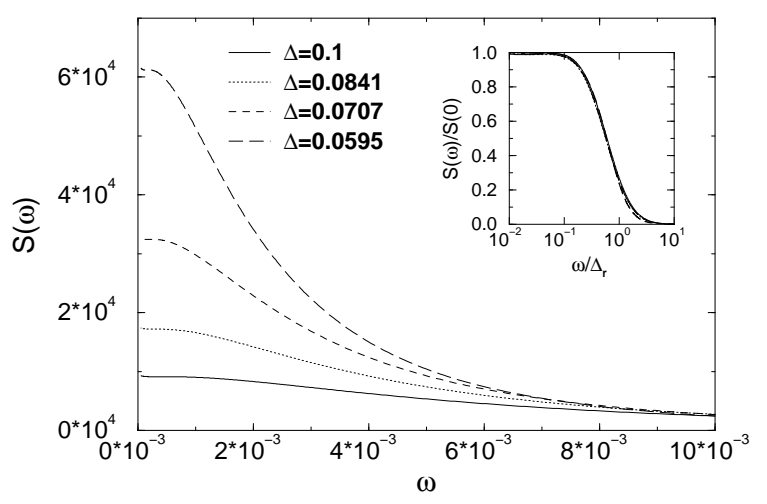

FIG. 18. Spin-spin correlation function $S(\omega)=2 C(\omega) / \omega^{\delta}$ at the Toulouse point $\alpha=\frac{1}{2}$ for the Ohmic case $s=1$ and various values of $\Delta$. The inset shows the scaling of these curves $\left(S(\omega) / S(0)\right.$ plotted versus $\left.\omega / \Delta_{\mathrm{r}}\right)$ together with the exact result (thick dashed line).

where $\delta$ is the exponent fitted to $C(\omega)$ in the small frequency regime.

In Fig. 17, $C(\omega)$ is shown for the Ohmic case and a set of $\alpha$ values close to the critical $\alpha_{\mathrm{c}}$. The spin-spin correlation function shows the expected power-law behavior, $C(\omega) \propto \omega$, in the low-frequency regime $\omega<T^{*}$. In the limit of $\alpha \rightarrow \alpha_{\mathrm{c}}, C(\omega)$ shows a divergence for $\omega>T^{*}$, $C(\omega) \propto \omega^{-1}$, with logarithmic corrections.

In Fig. 18, $S(\omega)$ is plotted at the Toulouse point $\left(\alpha=\frac{1}{2}\right)$ of the Ohmic spin-boson model for several values of $\Delta$. At this point, the Ohmic spin-boson model is exactly solvable, as discussed in Ref. 15. In the inset of Fig. 18, all the curves are rescaled onto one curve with a renormalized tunneling amplitude $\Delta_{\mathrm{r}}$. Here, $\Delta_{\mathrm{r}}$ is defined as

$$
\Delta_{\mathrm{r}, \mathrm{NRG}}=\frac{\chi_{\mathrm{e}} \Delta_{\mathrm{r}, \mathrm{e}}}{\chi_{\mathrm{NRG}}}
$$

where $\chi_{\mathrm{e}}$ is the exact susceptibility and $\Delta_{\mathrm{r}, \mathrm{e}}$ the exact renormalized tunnelling amplitude at the Toulouse point:

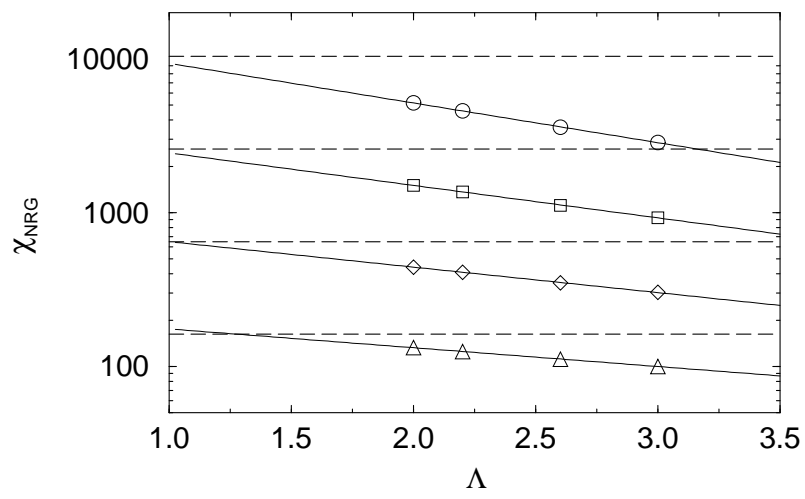

FIG. 19. NRG results for $\chi_{\mathrm{NRG}}$ calculated at the Toulouse point $\alpha=\frac{1}{2}$ for the Ohmic case $s=1$ and various values of $\Lambda$ and $\Delta$ (circles: $\Delta=0.0125$, squares: $\Delta=0.025$, diamonds: $\Delta=0.05$, triangles: $\Delta=0.1)$. Dashed lines show the exact values $\chi_{\mathrm{e}}=16 /\left(\pi^{2} \Delta^{2}\right)$ and thin solid lines are fits to the numerical results.

$\Delta_{\mathrm{r}, \mathrm{e}}=\pi \Delta^{2} / 2$ and $\chi_{\mathrm{e}} \Delta_{\mathrm{r}, \mathrm{e}}=8 / \pi$. The quantity $\chi_{\mathrm{NRG}}$ is the susceptibility calculated from the NRG, eq. (67). The comparison of the result from the bosonic NRG with the exact rescaled $S(\omega) / S(0)$ shows good agreement (see the inset of Fig. 18). The exact result is given by ${ }^{15}$ :

$$
\frac{S(\omega)}{S(0)}=\frac{1}{8\left(1+x^{2}\right)}\left[\frac{\ln \left(1+4 x^{2}\right)}{x^{2}}+\frac{2 \arctan (2 x)}{x}\right] \text {, }
$$

with $x=\frac{\omega}{\Delta_{\mathrm{r}, \mathrm{e}}}$.

The NRG results for $\chi_{\mathrm{NRG}}$ deviate significantly from the exact value $\chi_{\mathrm{e}}=16 /\left(\pi^{2} \Delta^{2}\right)$. However, as shown in Fig. 19, this deviation is entirely due to discretization effects and the extrapolation $\Lambda \rightarrow 1$ shows almost perfect agreement with the exact result. Note that the exact value for $\chi_{\mathrm{e}}$ has been obtained for a soft cut-off in the bath spectral function, $J(\omega)=2 \pi \alpha \omega \exp \left(-\omega / \omega_{\mathrm{c}}\right)$. To allow for a comparison, the logarithmic discretization has to be performed for the same soft cut-off (we introduce a high-energy hard cut-off at $\omega=15 \omega_{\mathrm{c}}$ ).

The scaling behavior of $S(\omega) / S(0)$ for fixed $\alpha$ and different values of $\Delta$ is shown in Fig. 20. For this we need to identify an energy scale $T^{*}$ as in Sec. III C. There are, as usual, various possibilities to define the energy scale: the position of the peak in $C(\omega), \omega_{\mathrm{p}}$, the quantity $1 / \chi$, and the $T^{*}$ as defined in eq. (51). Obviously, we have $T^{*} \propto \omega_{\mathrm{p}} \propto 1 / \chi \propto \Delta_{\mathrm{r}}$, and we choose $\Delta_{\mathrm{r}}=8 /(\pi \chi)$ for the energy scale in Fig. 20. The scaling curves shown in Fig. 20 are in good agreement with the ones calculated in Ref. 47; in particular, we find that the coherent peak in $S(\omega)$ disappears when $\alpha$ is larger than $\alpha^{*} \approx 0.3$.

In our notation, the Shiba relation reads ${ }^{41}$

$$
2 \alpha\left(\frac{\chi}{2}\right)^{2}=S(0) \text {. }
$$

Table I shows the results from the bosonic NRG for the Ohmic case and various values of $\alpha$ and $\Delta$. The parameter $\delta$ is the exponent defined in eq. (68). We find that the Shiba relation is fulfilled within an error of about $10 \%$. 


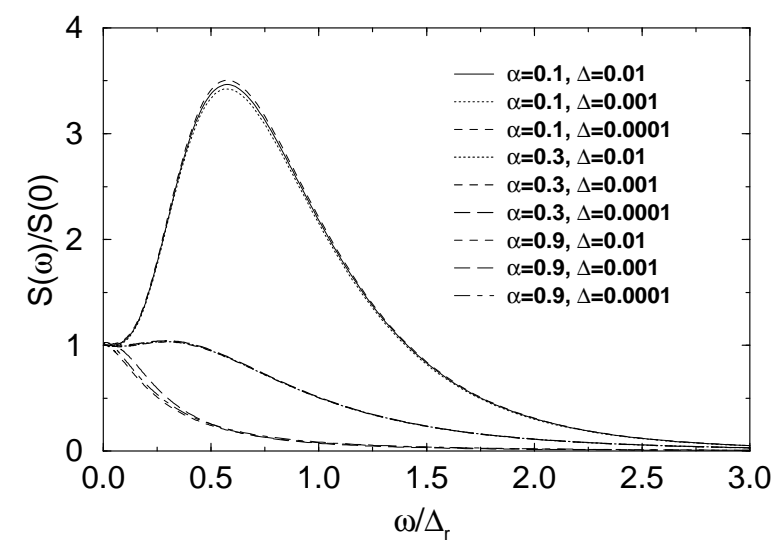

FIG. 20. Scaling spectra for the spin-spin correlation function $S(\omega)=2 C(\omega) / \omega^{\delta}$ for various values of $\alpha$ in the Ohmic case $s=1$.

TABLE I. Results from the bosonic NRG for the Shiba-relation in the Ohmic case for various values of $\alpha$ and $\Delta$.

\begin{tabular}{ccccccc}
\hline \hline$s$ & $\alpha$ & $\Delta$ & $\delta$ & $2 \alpha\left(\frac{\chi}{2}\right)^{2}$ & $S(0)$ & \%error \\
1.0 & 0.02 & 0.005 & 1.018 & $0.201 \times 10^{4}$ & $0.221 \times 10^{4}$ & $9.9 \%$ \\
1.0 & 0.1 & 0.01 & 1.018 & $0.603 \times 10^{4}$ & $0.631 \times 10^{4}$ & $4.6 \%$ \\
1.0 & 0.4 & 0.01 & 1.018 & $0.294 \times 10^{7}$ & $0.308 \times 10^{7}$ & $4.9 \%$ \\
1.0 & 0.5 & 0.025 & 1.018 & $0.154 \times 10^{7}$ & $0.163 \times 10^{7}$ & $5.8 \%$ \\
1.0 & 0.7 & 0.03 & 1.019 & $0.376 \times 10^{9}$ & $0.416 \times 10^{9}$ & $10.6 \%$ \\
1.0 & 0.9 & 0.1 & 1.018 & $0.172 \times 10^{10}$ & $0.192 \times 10^{10}$ & $11.6 \%$ \\
\hline \hline
\end{tabular}

\section{B. Order Parameter}

In the localized phase, which corresponds to the ordered phase of the $1 / r^{2}$ Ising model, it is straightforward to define an order parameter, $m$, corresponding to the magnetization of the Ising model. In the language of the spin-boson model, it corresponds to the static, i.e., $\omega=0$, part of the spin autocorrelation function; in the language of the anisotropic Kondo model this just measures the prefactor of the Curie part of the local susceptibility, i.e., the unscreened fraction of the impurity moment. The Kosterlitz-Thouless nature of the transition implies a jump of the order parameter at the phase transition.

We extract this order parameter from the $\delta(\omega)$ contribution to $C(\omega)$. The identification of such a $\delta$-peak in the spectrum of $C(\omega)$ requires some extra care. The spectrum calculated with the NRG consists of $\delta$-peaks only, which have to be broadened suitably to give spectra as shown, for example, in Fig. 17. Therefore, one has to decide whether a $\delta$-peak in the spectrum belongs to the continuum or whether it survives as a $\delta$-peak in the thermodynamic limit ${ }^{48}$. The procedure is illustrated in Fig. 21. Let us first note that the matrix element $\left|\left\langle 0\left|\sigma_{z}\right| 0\right\rangle\right|^{2}$ van-

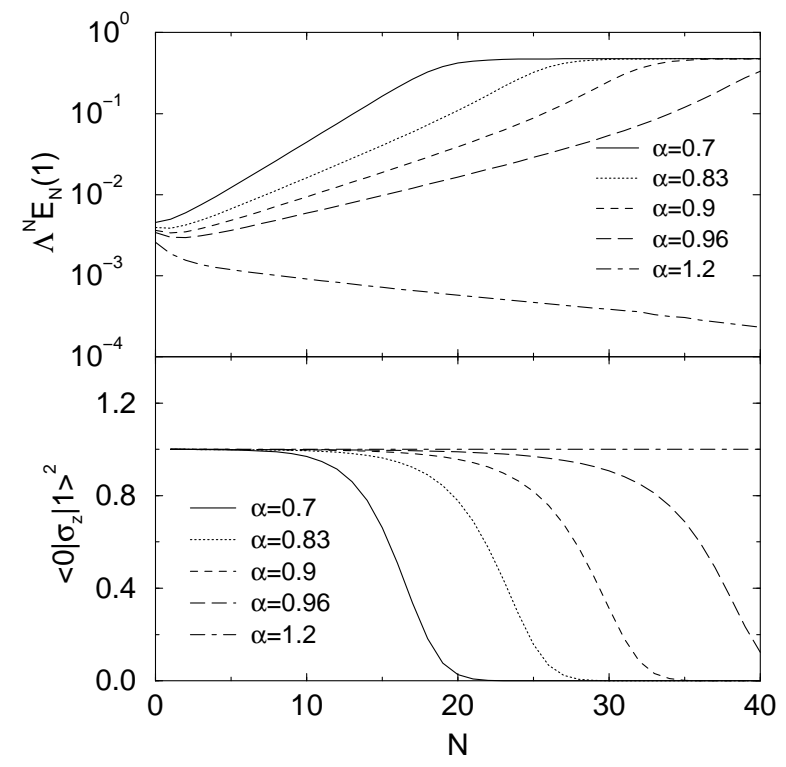

FIG. 21. (a) Energy of the first excited state $E(1)$ versus iteration number for $s=1.0, \Delta=0.01$, and various values of $\alpha$. (b) matrix element $\left|\left\langle 0\left|\sigma_{z}\right| 1\right\rangle\right|^{2}$ versus iteration number for the same parameters as in (a).

ishes for all parameters of Fig. 21. We therefore plot the matrix element $\left|\left\langle 0\left|\sigma_{z}\right| 1\right\rangle\right|^{2}$ in Fig. 21b together with the energy $E(1)$ in Fig. 21a. We observe that for $\alpha>\alpha_{\mathrm{c}}$ the energy $E(1)$ vanishes faster than $\Lambda^{-N}$ with increasing iteration number $N$, whereas the matrix element approaches a constant, $\left|\left\langle 0\left|\sigma_{z}\right| 1\right\rangle\right|^{2} \rightarrow$ const $\approx 1$. In the thermodynamic limit, this gives the $\delta$-peak at $\omega=0$, with the weight given by the matrix element $\left|\left\langle 0\left|\sigma_{z}\right| 1\right\rangle\right|^{2}$ which corresponds to the order parameter $m$. On the other hand, for $\alpha<\alpha_{\mathrm{c}}$ the energy $E(1)$ is proportional to $\Lambda^{-N}$, and the corresponding $\delta$-peak is therefore interpreted as being part of the continuum.

These arguments result in an order parameter $m(\alpha)$ which is zero for $\alpha<\alpha_{\mathrm{c}}$ and jumps to a finite value for $\alpha \geq \alpha_{\mathrm{c}}$. In the sub-Ohmic case, the order parameter shows power-law behavior near the quantum phase transition, which will be discussed in detail elsewhere. As an aside, we note that the order parameter can also be extracted from the Curie part of the static local susceptibility $\chi(T)$.

\section{CONCLUSIONS}

In this paper we have discussed a generalization of Wilson's NRG technique to quantum impurity problems with a bosonic bath. Focussing on the application to the spinboson model, we have shown that this novel method provides reliable results for both static and dynamic quantities in the whole range of model parameters and temperatures. For the case of Ohmic damping, we have compared our data to existing results in the literature and found good agreement. The bosonic NRG is able to reproduce 
the expected scaling behavior as function of temperature or frequency. For sub-Ohmic damping, there is a line of continuous boundary quantum phase transitions for all $0<s<1$, with exponents varying as function of $s$; details of the associated quantum critical behavior will be discussed in a forthcoming paper (see also Refs. 13, 31).

We have outlined several details of the numerical implementation of the bosonic NRG. Two general strategies were discussed, termed chain-NRG and star-NRG: both use a sequence of boson states with exponentially decreasing energy scales, but in the chain-NRG the bath states form a chain and the impurity couples to the first chain site only, whereas in the star-NRG the impurity is coupled to all bath sites which are not connected to each other. The advantages and disadvantages of both methods were discussed in detail, together with the important issue of the optimal choice of a basis set of bosonic states at each bath site. This problem is inherent to the bosonic NRG, as the infinite Hilbert space has to be truncated, and specific solutions have to be found for the problem at hand. We have argued that in the Ohmic, super-Ohmic and sub-Ohmic cases of the spin-boson model (except for the flow to the localized fixed point in the latter case) the basis formed by the lowest boson number eigenstates is sufficient, and all fixed points are properly captured in the NRG. Most of the results in this paper were obtained with this basis choice using the chain-NRG method; we have given a detailed account on convergence issues with respect to truncation and discretization parameters. In the sub-Ohmic case, the boson numbers diverge in the localized regime in the low-energy limit, and a different basis choice is needed. We have described suitable basis states using displaced harmonic oscillators, which solve the problem for the star-NRG. Open numerical issues include a reliable implementation of the displaced-oscillator basis for the chain-NRG, a more accurate calculation of dynamic quantities, as well as the numerical stability for very long iterations, i.e., very small energy scales.

The bosonic NRG can be easily generalized to impurities with multiple bosonic baths or both fermionic and bosonic baths. This is the subject of current work and will allow the study of large classes of impurity models, e.g., so-called Bose Kondo ${ }^{21}$ and BoseFermi Kondo models ${ }^{49}$. These models are known to display intermediate-coupling fixed points associated with universal local-moment fluctuations. The Bose-Fermi Kondo model arises in the context of extended dynamical mean-field theory $(\mathrm{EDMFT})^{50}$, where a lattice model is mapped onto an impurity model with a fermionic bath (representing conduction electrons) and a bosonic bath (representing bulk spin fluctuations). The quantum phase transition appearing in the Bose-Fermi Kondo model has been proposed to describe local quantum critical behavior in EDMFT, which may be relevant to the physics of certain heavy-fermion quantum phase transitions. However, a full numerical solution of the EDMFT equations at $T=0$ has not been presented to date, due to the lack of suitable impurity solvers. A version of the bosonic NRG may help to overcome this difficulty.

Other applications of the bosonic NRG can likely be found in the rapidly developing field of ultracold bosonic gases, where indeed various realizations of spin-boson physics have been proposed ${ }^{51}$.

Further, the physics of decoherence of qubits naturally leads to variants of the spin-boson model. Interestingly, the description of $1 / f$ noise in electrical circuits leads to sub-Ohmic damping with $s=0$ (at least over a certain range of energies). In this sub-Ohmic parameter regime, the bosonic NRG is one of the few methods which can give reliable answers, including, e.g., the existence of a quantum phase transition for $0<s<1-$ note that this transition does not appear in the popular non-interacting blip (NIBA) approximation ${ }^{15}$. Other modifications of standard spin-boson physics include the influence of localized modes which interact with the qubit of interest those modes can be represented by a discrete spin system, leading to so-called central spin models ${ }^{17}$. Usually, such systems map onto spin-boson models with a spectral density consisting of a continuous (e.g. Ohmic) background and sharp peaks at certain frequencies ${ }^{16}$; these models can be easily studied using NRG.

\section{ACKNOWLEDGMENTS}

We thank M. Garst, T.-H. Gimm, H. Horner, E. Jeckelmann, S. Kehrein, Th. Pruschke, A. Rosch, P. Wölfle, and W. Zwerger for discussions. This research was supported by the DFG through SFB 484 (RB, HJL) and the Center for Functional Nanostructures Karlsruhe (MV), and by the Alexander von Humboldt foundation (NHT).

\section{APPENDIX A: CALCULATION OF THE PARAMETERS OF THE SEMI-INFINITE CHAIN}

In this appendix, we describe the orthogonal transformation from the star-Hamiltonian (11) to the semiinfinite chain form [the chain-Hamiltonian (14)] and present equations relating the parameters of the two Hamiltonians.

We start from the star-Hamiltonian (11):

$$
H_{\mathrm{s}}=H_{\mathrm{loc}}+\sum_{n=0}^{\infty} \xi_{n} a_{n}^{\dagger} a_{n}+\frac{\sigma_{z}}{2 \sqrt{\pi}} \sum_{n=0}^{\infty} \gamma_{n}\left(a_{n}+a_{n}^{\dagger}\right)
$$

Our goal is to transform it to a semi-infinite chain eq. (14):

$$
\begin{aligned}
H_{\mathrm{c}} & =H_{\mathrm{loc}}+\sqrt{\frac{\eta_{0}}{\pi}} \frac{\sigma_{z}}{2}\left(b_{0}+b_{0}^{\dagger}\right) \\
& +\sum_{n=0}^{\infty}\left[\epsilon_{n} b_{n}^{\dagger} b_{n}+t_{n}\left(b_{n}^{\dagger} b_{n+1}+b_{n+1}^{\dagger} b_{n}\right)\right] .
\end{aligned}
$$


Here the main difference to the fermionic case (such as the Kondo model studied in Ref. 1) is that the bosonic spectral function $J(\omega)$ is restricted to positive frequencies only. This asymmetry of $J(\omega)$ influences the structure of the semi-infinite chain Hamiltonian (additional on-site energies $\epsilon_{n}$ appear in eq. (A2) which are not present for particle-hole symmetry in the fermionic case).

In the following we define a real orthogonal transformation $U$,

$$
b_{n}=\sum_{m=0}^{\infty} U_{n m} a_{m}
$$

with $U^{T} U=U U^{T}=1, U^{*}=U$, so that the inverse transformation reads

$$
a_{n}=\sum_{m=0}^{\infty} U_{m n} b_{m}
$$

Comparing the coupling terms between spin and bosons in $H_{\mathrm{s}}$ and $H_{\mathrm{c}}$ gives

$$
b_{0}=\frac{1}{\sqrt{\eta_{0}}} \sum_{n=0}^{\infty} \gamma_{n} a_{n}
$$

so that

$$
U_{0 n}=\frac{\gamma_{n}}{\sqrt{\eta_{0}}}
$$

The bosonic commutation relation $\left[b_{0}, b_{0}^{\dagger}\right]=1$ applied to eq. (A5) gives

$$
\eta_{0}=\sum_{n=0}^{\infty} \gamma_{n}^{2}=\int_{0}^{\omega_{c}} J(x) \mathrm{d} x
$$

We are left with the equivalence of the free bosonic part in $H_{\mathrm{s}}$ and $H_{\mathrm{c}}$ :

$$
\sum_{n=0}^{\infty} \xi_{n} a_{n}^{\dagger} a_{n}=\sum_{n=0}^{\infty}\left[\epsilon_{n} b_{n}^{\dagger} b_{n}+t_{n}\left(b_{n}^{\dagger} b_{n+1}+b_{n+1}^{\dagger} b_{n}\right)\right] .
$$

To obtain the recursion relations for $\epsilon_{n}$ and $t_{n}$, we first put eq. (A4) into the left-hand side of eq. (A8) (for the annihilation operators only). We then sort the resulting equation for the operators $b_{m}$. Comparing the prefactors of the terms containing $b_{m}$ we obtain for the operator $b_{0}$ :

$$
\sum_{n=0}^{\infty} \xi_{n} a_{n}^{\dagger} U_{0 n}=\epsilon_{0} b_{0}^{\dagger}+t_{0} b_{1}^{\dagger}
$$

and for $b_{m}$ with $m>0$ :

$$
\sum_{n=0}^{\infty} \xi_{n} a_{n}^{\dagger} U_{m n}=\epsilon_{m} b_{m}^{\dagger}+t_{m} b_{m+1}^{\dagger}+t_{m-1} b_{m-1}^{\dagger} .
$$

The expression for $\epsilon_{0}$ can be obtained from taking the commutator between $b_{0}$ and eq. (A9):

$$
\epsilon_{0}=\sum_{n=0}^{\infty} \xi_{n} U_{0 n}^{2}
$$

From eq. (A9), we also obtain

$$
t_{0} b_{1}^{\dagger}=\sum_{n=0}^{\infty}\left(\xi_{n}-\epsilon_{0}\right) U_{0 n} a_{n}^{\dagger}
$$

which gives immediately

$$
U_{1 n}=\frac{1}{t_{0}}\left(\xi_{n}-\epsilon_{0}\right) U_{0 n} .
$$

The value of $t_{0}$ can be calculated by taking the commutator with the corresponding adjoint operator on both sides of eq. (A12). This results in

$$
t_{0}=\frac{1}{\sqrt{\eta_{0}}}\left[\sum_{n=0}^{\infty}\left(\xi_{n}-\epsilon_{0}\right)^{2} \gamma_{n}^{2}\right]^{\frac{1}{2}}
$$

Equations (A6), (A11), (A13), and (A14) initialize the recursion relations for the calculation of $\epsilon_{m}, t_{m}$ and $U_{m n}$. These recursion relations can be obtained by starting with eq. (A10) and proceeding in a similar way as above.

The commutator beween $b_{m}$ and eq. (A10) gives

$$
\epsilon_{m}=\sum_{n=0}^{\infty} \xi_{n} U_{m n}^{2}
$$

From eq. (A10) we also find

$$
t_{m} b_{m+1}^{\dagger}=\sum_{n=0}^{\infty}\left(\xi_{n} U_{m n}-\epsilon_{m} U_{m n}-t_{m-1} U_{m-1 n}\right) a_{n}^{\dagger} .
$$

From this equation, we obtain the expression for $U_{m+1 n}$ :

$$
U_{m+1 n}=\frac{1}{t_{m}}\left[\left(\xi_{n}-\epsilon_{m}\right) U_{m n}-t_{m-1} U_{m-1 n}\right] .
$$

The values of $t_{m}$ can be calculated by taking the commutator with the corresponding adjoint operator on both sides of eq. (A16). This results in

$$
t_{m}=\left[\sum_{n=0}^{\infty}\left[\left(\xi_{n}-\epsilon_{m}\right) U_{m n}-t_{m-1} U_{m-1 n}\right]^{2}\right]^{\frac{1}{2}}
$$

Equations (A15), (A17), and (A18) complete the recursion relations for the calculation of the parameters of the chain Hamiltonian (14).

Despite the simple structure of the input spectral function, $J(\omega)=2 \pi \alpha \omega^{s}(s \geq 0)$, we did not succeed in solving the recursion relations analytically (this is in fact 
possible for the particle-hole symmetric soft-gap Anderson model, where the hybridization function vanishes at the Fermi level as $\Delta(\omega)=\Delta_{0}|\omega|^{r}$, see Ref. 32; due to the particle-hole symmetry, the $\epsilon_{n}$ vanish and the recursion relations have a much simpler structure). Therefore the recursion relations have to be iterated numerically, in a similar way as for the fermionic case. Note that the derivation of the chain-Hamiltonian in the asymmetric fermionic case, where $\epsilon_{n} \neq 0$, is very similar to the bosonic case described above. The only differences are the structure of the coupling between impurity and the bath, and the fact that all commutators have to be replaced by anticommutators. For a recent application of the NRG to a fermionic model with an asymmetric hybridization function, see Ref. 52 .

The resulting parameters of the chain-Hamiltonian, $\epsilon_{n}$ and $t_{n}$, both fall off as $\Lambda^{-n}$; in contrast to the fermionic case where $t_{n} \propto \Lambda^{-n / 2}$. For large $n$, the ratio $t_{n} / \epsilon_{n}$ approaches an $s$-dependent value.

\section{APPENDIX B: OPTIMAL BOSONIC BASIS IN THE STAR-NRG}

In this appendix, we present details of how we implement the optimal basis for the bosons in the star-NRG to overcome the problem of the boson number divergence when the flow is to the localized fixed point (see the discussions in Secs. IIE and IIID).

In each step of the star-NRG, a new bosonic degree of freedom is added to the Hamiltonian. The renormalization group transformation is given by eq. (19):

$$
\begin{aligned}
& H_{N+1, \mathrm{~s}}=\Lambda H_{N, \mathrm{~s}} \\
+ & \Lambda^{N+1}\left[\xi_{N+1} a_{N+1}^{\dagger} a_{N+1}+\frac{\sigma_{z}}{2 \sqrt{\pi}} \gamma_{N+1}\left(a_{N+1}+a_{N+1}^{\dagger}\right)\right]
\end{aligned}
$$

The problem discussed in Sec. IIE is that upon approaching the localized fixed point in the sub-Ohmic case, the displacements $\theta_{N}$ for the bosonic site $N$ increase exponentially with $N$, see eq. (36). The displacements can be taken into account by constructing an appropriate basis $|s(N+1)\rangle$ for the new bosonic degree of freedom.

To construct this basis, we start with a simplified Hamiltonian of the form:

$$
\bar{H}=a^{\dagger} a+\theta \sigma_{z}\left(a^{\dagger}+a\right),
$$

and proceed as follows: in the first step, we set up an optimized basis for $\bar{H}$ (optimized in the sense that the lowest lying eigenstates of $\bar{H}$ are described with only a small number of basis states). Then we use this basis, denoted as $|s(N+1)\rangle_{\theta}$, for the actual NRG iteration, and finally, we describe a self-consistent procedure to determine the parameter $\theta$.

Let us first discuss how to construct the optimized basis for $\bar{H}$. Consider the following operators:

$$
H_{ \pm \theta}=a^{\dagger} a \pm \theta\left(a^{\dagger}+a\right) .
$$

The eigenstates of $H_{ \pm \theta}$ are denoted as $|m\rangle_{ \pm \theta}(m=$ $0,1, \ldots)$. We obtain

$$
H_{ \pm \theta}|m\rangle_{ \pm \theta}=\left(m-\theta^{2}\right)|m\rangle_{ \pm \theta},
$$

and

$$
|m\rangle_{ \pm \theta}=e^{\mp \theta\left(a^{\dagger}-a\right)}|m\rangle,
$$

with $|m\rangle$ the eigenstates of $a^{\dagger} a$. The basis states should describe the $+\theta$ and $-\theta$ displacements on an equal footing; therefore we proceed with symmetrized eigenstates $|m\rangle_{\mathrm{e} / \mathrm{o}}$ constructed in the following way:

$$
\begin{array}{r}
|m\rangle_{\mathrm{e}}=c_{\mathrm{e}, m}\left[|m\rangle_{\theta}+(-1)^{m}|m\rangle_{-\theta}\right] \\
|m\rangle_{\mathrm{o}}=c_{\mathrm{o}, m}\left[|m\rangle_{\theta}-(-1)^{m}|m\rangle_{-\theta}\right] \\
m=0,1, \ldots, \frac{N_{\mathrm{b}}}{2}-1,
\end{array}
$$

with normalization constants $c_{\mathrm{e} / \mathrm{o}, m}$. Note that here we have to choose an even number $N_{\mathrm{b}}$. The even and odd parity states are orthogonal to each other, ${ }_{\mathrm{e}}\langle n \mid m\rangle_{\mathrm{o}}=0$, whereas states with the same parity are not necessarily orthogonal. An orthogonalization procedure for both even and odd parity states then gives the final set of basis states:

$$
\begin{aligned}
|\overline{0}\rangle_{\mathrm{e}} & =|0\rangle_{\mathrm{e}} \\
|\overline{1}\rangle_{\mathrm{e}} & =C_{\mathrm{e}, 1}\left\{|1\rangle_{\mathrm{e}}-\mathrm{e}_{\mathrm{e}}\langle\overline{0} \mid 1\rangle_{\mathrm{e}}|\overline{0}\rangle_{\mathrm{e}}\right\} \\
|\overline{2}\rangle_{\mathrm{e}} & =C_{\mathrm{e}, 2}\left\{|2\rangle_{\mathrm{e}}-\mathrm{e}_{\mathrm{e}}\langle\overline{1} \mid 2\rangle_{\mathrm{e}}|\overline{1}\rangle_{\mathrm{e}}-{ }_{\mathrm{e}}\langle\overline{0} \mid 2\rangle_{\mathrm{e}}|\overline{0}\rangle_{\mathrm{e}}\right\} \\
\ldots &
\end{aligned}
$$

with normalization constants $C_{\mathrm{e} / \mathrm{o}, m}$. The same orthogonalization is performed for the odd parity states. In this way, we obtain $N_{\mathrm{b}}$ orthogonal states, characterized by the parameter $\theta$, which form the basis $|s(N+1)\rangle_{\theta}$ for the diagonalization of $H_{N+1, \mathrm{~s}}$ :

$$
|s(N+1)\rangle_{\theta}=\left\{|\overline{0}\rangle_{\mathrm{e}},|\overline{1}\rangle_{\mathrm{e}}, \ldots,|\overline{0}\rangle_{\mathrm{o}},|\overline{1}\rangle_{\mathrm{o}}, \ldots\right\} .
$$

The calculation of the matrix elements $H_{N+1, \mathrm{~s}}\left(r s, r^{\prime} s^{\prime}\right)$ (see eqs. (43-46)) involves matrix elements of the form

$$
{ }_{\theta}\left\langle s(N+1)\left|a_{N+1}+a_{N+1}^{\dagger}\right| s^{\prime}(N+1)\right\rangle_{\theta},
$$

To evaluate these matrix elements [and the scalar products in eq. (B7)] we have to express the states $|\bar{m}\rangle_{\mathrm{e} / \mathrm{o}}$ in terms of the eigenstates $|n\rangle$ of $a_{N+1}^{\dagger} a_{N+1}$. This can be performed using the following recursion relations for $\langle n \mid m\rangle_{\theta}$ :

$$
\begin{aligned}
\langle n \mid m+1\rangle_{\theta} & =\frac{\theta}{\sqrt{m+1}}\langle n \mid m\rangle_{\theta}+\frac{\sqrt{n}}{\sqrt{m+1}}\langle n-1 \mid m\rangle_{\theta}, \\
\langle n \mid 0\rangle_{\theta} & =\frac{(-\theta)^{n}}{\sqrt{n !}} e^{-\frac{1}{2} \theta^{2}} \\
\langle 0 \mid m\rangle_{\theta} & =\frac{\theta^{m}}{\sqrt{m !}} e^{-\frac{1}{2} \theta^{2}}
\end{aligned}
$$


(and for $\langle n \mid m\rangle_{-\theta}$ by replacing $\theta$ by $-\theta$ ). The summation over $n$ in the calculation of matrix elements and scalar products has to be performed numerically which limits the number of states $|n\rangle$ to some finite, although very large, value $L$ (values up to $L \approx 10^{7}$ can be used). To construct an optimized basis for the displacements $\theta, L$ should be large enough (at least of the order of $\theta^{2}$ ) to include a sufficient number of states $|n\rangle$ in the calculation.

For the special case of $\Delta=0$, the parameter $\theta$ for the construction of the basis $|s(N+1)\rangle_{\theta}$ is exactly known (see Sec. II E). This is different in the general case of finite $\Delta$ where we have to find a scheme to determine the optimal value $\theta^{*}$. The general strategy to find this optimal value has been discussed in Sec. II F. For the actual numerical calculation it turns out that the following self-consistent scheme is much more efficient.

For the ground state $|g\rangle$ of $\bar{H}$ (B2) the expectation value $\left\langle g\left|a^{\dagger} a\right| g\right\rangle$ is equal to $\theta^{2}$. We use this relation to determine the $\theta$ used for the NRG calculation:

$$
\theta=\sqrt{{ }_{N+1}\left\langle g\left|a_{N+1}^{\dagger} a_{N+1}\right| g\right\rangle_{N+1}},
$$

where $|g\rangle_{N+1}$ is the ground state of $H_{N+1, \mathrm{~s}}$ which has been obtained from diagonalizing the matrix $H_{N+1, \mathrm{~s}}\left(r s, r^{\prime} s^{\prime}\right)$ using the basis $|s(N+1)\rangle_{\theta}$ characterized by the parameter $\theta$. In other words, eq. (B11) defines a self-consistent scheme to calculate $\theta$ for each NRG step.

The converged value $\theta^{*}$ gives the optimal basis for adding the site $N+1$ in the NRG iteration. It corresponds to the value $\theta^{*}$ which characterizes the minimum of the energy levels in Fig. 4. The energy levels calculated in this way show a much weaker dependence on $N_{\mathrm{b}}$ which leads, for example, to the rapid convergence of $n_{\mathrm{b}}$ with increasing $N_{\mathrm{b}}$ as shown in Fig. 13.

After the diagonalization of $H_{N+1, \mathrm{~s}}$ with the optimized basis $|s(N+1)\rangle_{\theta^{*}}$, we can continue the NRG iteration by adding the site $N+2$.

${ }^{1}$ K. G. Wilson, Rev. Mod. Phys. 47, 773 (1975).

${ }^{2}$ H. R. Krishna-murthy, J. W. Wilkins, and K. G. Wilson, Phys. Rev. B 21, 1003 (1980); ibid. 21, 1044 (1980).

${ }^{3}$ A. C. Hewson, The Kondo Problem to Heavy Fermions (Cambridge Univ. Press, Cambridge 1993).

${ }^{4}$ T. A. Costi, in Density-Matrix Renormalization - A New Numerical Method in Physics, Eds. I. Peschel et al. (Springer 1999).

${ }^{5}$ R. Bulla, Adv. Solid State Phys. 40, 169 (2000).

${ }^{6}$ O. Sakai and Y. Shimizu, J. Phys. Soc. Jpn. 61, 2333 (1992); ibid. 61, 2348 (1992); O. Sakai, Y. Shimizu, and N. Kaneko, Physica B 186-188, 323 (1993).

${ }^{7}$ K. Ingersent and B. A. Jones, Physica B 199-200, 402 (1994).

${ }^{8}$ A. C. Hewson and D. Meyer, J. Phys. Condens. Matter 14,
427 (2002); D. Meyer, A. C. Hewson, and R. Bulla, Phys. Rev. Lett. 89, 196401 (2002).

${ }^{9}$ W. Metzner and D. Vollhardt, Phys. Rev. Lett. 62, 324 (1989).

10 A. Georges, G. Kotliar, W. Krauth, and M. J. Rozenberg, Rev. Mod. Phys. 68, 13 (1996).

${ }^{11}$ R. Bulla, Phys. Rev. Lett. 83, 136 (1999).

${ }^{12}$ R. Bulla, T. A. Costi, and D. Vollhardt, Phys. Rev. B 64, 045103 (2001).

${ }^{13}$ R. Bulla, N. Tong, and M. Vojta, Phys. Rev. Lett. 91, 170601 (2003).

${ }^{14}$ A. J. Leggett, S. Chakravarty, A. T. Dorsey, M. P. A. Fisher, A. Garg, and W. Zwerger, Rev. Mod. Phys. 59, 1 (1987).

${ }^{15}$ U. Weiss, Quantum dissipative systems, 2nd ed. (World Scientific, Singapore, 1999).

${ }^{16}$ S. Kleff, S. Kehrein, and J. von Delft, Physica E 18, 343 (2003); F. K. Wilhelm, S. Kleff, and J. von Delft, Chem. Phys. 296, 345 (2004).

${ }^{17}$ N. Prokovév and P. C. E. Stamp, Rep. Prog. Phys. 63, 669 (2000); P. C. E. Stamp and I. S. Tupitsyn, Chem. Phys. 296, 281 (2004).

18 T. A. Costi and R. H. McKenzie, Phys. Rev. A 68, 034301 (2003).

19 D. V. Khveshchenko, Phys. Rev. B 69, 153311 (2004).

${ }^{20}$ M. Thorwart and P. Hänggi, Phys. Rev. A 65, 012309 (2002); M. J. Storcz and F. K. Wilhelm, ibid. 67, 042319 (2003).

21 S. Sachdev, C. Buragohain, and M. Vojta, Science 286, 2479 (1999); A. H. Castro Neto, E. Novais, L. Borda, G. Zaránd, and I. Affleck, Phys. Rev. Lett. 91, 096401 (2003).

${ }^{22}$ A. Garg, J. N. Onuchic, and V. Ambegaokar, J. Chem. Phys. 83, 4491 (1985).

${ }^{23}$ L. Mühlbacher and R. Egger, J. Chem. Phys. 118, 179 (2003); Chem. Phys. 296, 193 (2004).

${ }^{24}$ R. Egger and C. H. Mak, Phys. Rev. B 50, 15210 (1994); K. Völker, Phys. Rev. B 58, 1862 (1998).

${ }^{25}$ Y. Nishiyama, Eur. Phys. J. B 12, 547 (1999).

26 T. A. Costi, Phys. Rev. Lett. 80, 1038 (1998).

${ }^{27}$ Both the sub-Ohmic spin-boson model and the pseudogap Kondo model display non-trivial quantum phase transitions, which are, however, in different universality classes, see: M. Vojta and L. Fritz, Phys. Rev. B 70, 094502 (2004); L. Fritz and M. Vojta, cond-mat/0408543.

28 S. Kehrein and A. Mielke, Phys. Lett. A 219, 313 (1996).

${ }^{29}$ H. Spohn and R. Dümcke, J. Stat. Phys. 41, 389 (1985).

30 J. M. Kosterlitz, Phys. Rev. Lett. 37, 1577 (1976).

${ }^{31}$ Results for the critical exponents in the sub-Ohmic case are shown in: M. Vojta, N.-H. Tong, and R. Bulla, condmat/0410132.

${ }^{32}$ R. Bulla, Th. Pruschke, and A. C. Hewson, J. Phys. Cond. Matter 9, 10463 (1997).

${ }^{33}$ For Jahn-Teller systems, the mapping to a bosonic chain was also considered in: S. N. Evangelou and A. C. Hewson, J. Phys. C 15, 7073 (1982).

34 A similar problem appears in DMRG calculations for the one-dimensional Holstein model, see: C. Zhang, E. Jeckelmann, and S. R. White, Phys. Rev. Lett. 80, 2661 (1998).

${ }^{35}$ P. W. Anderson, G. Yuval, and D. R. Hamann, Phys. Rev. B 1, 4464 (1970). 
${ }^{36}$ R. Bulla, M. T. Glossop, D. E. Logan, and Th. Pruschke. J. Phys.: Condens. Matter 12, 4899 (2000).

${ }^{37}$ W. C. Oliveira and L. N. Oliveira, Phys. Rev. B 49, 11986 (1994).

38 The relation $T^{*} \propto \Delta^{1 /\left(\alpha_{c}-\alpha\right)}$ (see Ref. 14) for the crossover scale in the Ohmic spin-boson model, obtained from various perturbative approaches, breaks down very close to $\alpha=1$, where it has to be replaced by $T^{*} \propto e^{\text {const } / \sqrt{\alpha_{c}-\alpha}}$, see: J. M. Kosterlitz, J. Phys. C 7, 1046 (1974). Our numerical data in Fig. 11 can be equivalently fitted with both forms, and the resulting values of $\alpha_{\mathrm{c}}$ differ by less than $2 \%$.

${ }^{39}$ T. A. Costi and G. Zaránd, Phys. Rev. B 59, 12398 (1999).

${ }^{40}$ R. Göhrlich and U. Weiss, Phys. Rev. B 38, 5245 (1998).

${ }^{41}$ M. Sassetti and U. Weiss, Phys. Rev. Lett. 65, 2262 (1990).

${ }^{42}$ R. Bulla and A. C. Hewson, Z. Phys. B 104, 333 (1997).

${ }^{43}$ H. O. Frota and L. N. Oliveira, Phys. Rev. B 33, 7871 (1986).

${ }^{44}$ O. Sakai, Y. Shimizu, and T. Kasuya, J. Phys. Soc. Jpn. 58, 3666 (1989).

${ }^{45}$ T. A. Costi and A. C. Hewson, Philos. Mag. B 65, 1165 (1992).

46 T. A. Costi, A. C. Hewson, and V. Zlatić, J. Phys.: Condens. Matter 6, 2519 (1994).

47 T. A. Costi and C. Kieffer, Phys. Rev. Lett. 76, 1683 (1996).

48 A $\delta(\omega)$ contribution to dynamic quantities has been discussed within the NRG for fermionic systems in S. C. Bradley, R. Bulla, A. C. Hewson, and G.-M. Zhang, Eur. Phys. J. B 11, 535 (1999); M. Vojta and R. Bulla, Phys. Rev. B 65, 014511 (2001).

49 A. M. Sengupta, Phys. Rev. B 61, 4041 (2000); L. Zhu and Q. Si, Phys. Rev. B 66, 024426 (2002); G. Zaránd and E. Demler, Phys. Rev. B 66, 024427 (2002); M. Kirćan and M. Vojta, Phys. Rev. B 69, 174421 (2004).

${ }^{50}$ Q. Si, S. Rabello, K. Ingersent, and J. L. Smith, Nature 413, 804 (2001); Phys. Rev. B 68, 115103 (2003).

${ }^{51}$ A. Recati, P. O. Fedichev, W. Zwerger, J. von Delft, and P. Zoller, cond-mat/0212413; cond-mat/0404533.

52 J. Martinek, M. Sindel, L. Borda, J. Barnas, R. Bulla, J. König, G. Schön, S. Maekawa, and J. von Delft, condmat/0406323. 